\title{
Special Section on Pharmacokinetic and Drug Metabolism Properties of Novel Therapeutic Modalities
}

\section{In Vitro Drug-Drug Interaction Evaluation of GaINAc Conjugated siRNAs Against CYP450 Enzymes and Transporters ${ }^{[]}$}

\author{
D Diane Ramsden, Jing-Tao Wu, Brad Zerler, Sajida lqbal, Jim Jiang, Valerie Clausen, \\ (D) Krishna Aluri, Yongli Gu, Sean Dennin, Joohwan Kim, and Saeho Chong \\ Alnylam Pharmaceuticals, Inc., Cambridge, Massachusetts (D.R., J.-T.W., J.J., V.C., K.A., Y.G., S.D., J.K., S.C.); The Medicines \\ Company, Parsippany, New Jersey (B.Z.); and Sanofi, Waltham, Massachusetts (S.I.)
}

Received March 8, 2019; accepted July 2, 2019

\section{ABSTRACT}

Small interfering RNAs (siRNAs) represent a new class of medicines that are smaller $(\sim 16,000 \mathrm{Da})$ than biologic therapeutics $(>150,000$ Da) but much larger than small molecules ( $<900 \mathrm{Da})$. Current regulatory guidance on drug-drug interactions (DDIs) from the European Medicines Agency, Food and Drug Administration, and Pharmaceutical and Medical Devices Agency provides no recommendations for oligonucleotide therapeutics including siRNAs; therefore, small molecule guidance documents have historically been applied. Over $\sim 10$ years, in vitro DDI investigations with siRNAs conjugated to a triantennary $\mathbf{N}$-acetylgalactosamine [(GaINAc)siRNA] ligand have been conducted during nonclinical drug development to elucidate the potential clinical DDI liability. GaINAc siRNAs were evaluated as substrates, inhibitors, or inducers of major cytochrome P450s (P450s) and as substrates and inhibitors of transporters. Aggregate analysis of these data demonstrates a low potential for DDI against P450s. Zero of five, 10, and seven are inducers, time-dependent inhibitors, or substrates, respectively, and nine of 12 do not inhibit any P450 isoform evaluated. Three GalNAc siRNAs inhibited CYP2C8 at supratherapeutic concentrations, and one mildly inhibited CYP2B6. The lowest $K_{\mathrm{i}}$ value of $28 \mu \mathrm{M}$ is $>3000$-fold above the therapeutic clinical $C_{\max }$ at steady state, and importantly no clinical inhibition was projected. Of four GaINAc
siRNAs tested none were substrates for transporters and one caused inhibition of P-glycoprotein, calculated not to be clinically relevant. The pharmacological basis for DDIs, including consideration of the target and/or off-target profiles for GalNAc siRNAs, should be made as part of the overall DDI risk assessment. If modulation of the target protein does not interfere with P450s or transporters, then in vitro or clinical investigations into the DDI potential of the GalNAc siRNAs are not warranted.

\section{SIGNIFICANCE STATEMENT}

Recommendations for evaluating DDI potential of small molecule drugs are well established; however, guidance for novel modalities, particularly oligonucleotide-based therapeutics are lacking. Given the paucity of published data in this field, in vitro DDI investigations are often conducted. The aggregate analysis of GalNAc-siRNA data reviewed herein demonstrates that, like new biological entities, these oligonucleotide-based therapeutic drugs are unlikely to result in DDIs; therefore, it is recommended that the need for in vitro or clinical investigations similarly be determined on a case-by-case basis. Given the mechanism of siRNA action, special consideration should be made in cases where there may be a pharmacological basis for DDIs.

\section{Introduction}

Harnessing the endogenous gene regulatory pathway of RNA interference is possible using chemically modified small interfering RNAs (siRNA) that induce short-term silencing of proteins and represent a new

https://doi.org/10.1124/dmd.119.087098.

S This article has supplemental material available at dmd.aspetjournals.org. class of drugs (Kim and Rossi, 2008). When introduced into cells, the guide (or antisense) strand of the siRNA loads into an enzyme complex called the RNA-induced silencing complex. This enzyme complex subsequently binds to its complementary mRNA sequence, mediating cleavage of the target mRNA and suppression of the target protein encoded by the mRNA (Elbashir et al., 2001). Unmodified siRNAs are rapidly degraded by extra- and intracellular nucleases, which limit tissue distribution upon systemic administration (Soutchek et al., 2004).

ABBREVIATIONS: ACN, acetonitrile; ASGPR, asialoglycoprotein receptor; ASO, antisense oligonucleotide; BCRP, breast cancer resistance protein; BSEP, bile salt export pump; $\mathrm{C}_{\mathrm{T}}$, threshold cycle; DDI, drug-drug interaction; EMA, European Medicines Agency; FDA, Food and Drug Administration; GalNAc, $\mathrm{N}$-acetylgalactosamine; HLM, human liver microsome; HMM, hepatocyte maintenance media; IS, internal standard; KH, Krebs-Henseleit; LC, liquid chromatography; LC-MS/MS, liquid chromatography-tandem mass spectrometry; MATE, multidrug and toxin extrusion protein; MS, mass spectrometry; OAT, organic anion transporter; OATP, organic anion transporting polypeptide; OCT, organic cation transporter; P450, cytochrome P450; P-gp, P-glycoprotein; rCYP, recombinant human cytochrome P450; RT, reverse transcription; siRNA, small interfering RNA; TOF, time-of-flight. 
Conjugation of the siRNA with $N$-acetylgalactosamine (GalNAc), which targets the asialoglycoprotein receptor (ASGPR), richly expressed on hepatocytes (Nair et al., 2014) has been successfully used as a delivery approach and leads to high levels of siRNA in hepatocytes. Multiple GalNAc siRNAs have achieved proof-of-concept status in the clinic (Fitzgerald et al., 2017; Pasi et al., 2017; Zimmermann et al., 2017). Current GalNAc siRNAs employ enhanced stabilization chemistry (Foster et al., 2018), a design that confers increased stability against nuclease degradation resulting in enhanced potency, lower dose, less frequent dosing, and reduced exposure to monomers (Janas et al., 2019). The enhanced stabilization chemistry and GalNAc delivery platform are used for several siRNA compounds currently in clinical development, including five that are in phase 3 development (givosiran, fitusiran, inclisiran, lumasiran, and vutrisiran), and one in phase 2 (cemdisiran).

There is no regulatory drug-drug interaction (DDI) guidance for oligonucleotide therapeutics; as such, they are considered to be small molecule drugs and evaluation of their potential for DDI is encouraged. To satisfy these recommendations, in vitro studies are typically carried out to evaluate whether a new chemical entity is a substrate of cytochrome P450s (P450s) or transporters, or if it can inhibit or induce major P450s. Since there are well-documented interactions due to inhibition of transporters, this is also generally evaluated in vitro during drug development. While there are some differences in the interpretation of a positive DDI signal depending on the regulatory authority (European Medicines Agency, 2012; Food and Drug Administration, 2017; Pharmaceutical and Medical Devices Agency, 2018), these agencies are united in the recommendation for conducting in vitro experiments to derive the kinetic parameters in order to contextualize the clinical potential for DDI. The European Medicines Agency (EMA) also specifically states that the potential for higher concentrations in the hepatocytes than in the plasma should be discussed, and if available data indicate that the drug may accumulate in hepatocytes, this should be considered in the area under the concentration-time curve ratio estimations (European Medicines Agency, 2012). These aspects are relevant for GalNAc siRNAs given their targeted delivery to the liver.

In 2018, the first siRNA therapeutic, ONPATTRO (patisiran), was approved. Patisiran is formulated as a lipid nanoparticle; therefore, the mode of delivery to hepatocytes is different from GalNAc-conjugated siRNAs, which are the focus of this paper. GalNAc siRNAs are $\sim 16,000 \mathrm{kDa}$, highly soluble, and negatively charged at physiologic $\mathrm{pH}$. They are rapidly taken up into the liver (target tissue) by ASGPR, where they are metabolized by endo- or exonucleases, followed by subsequent excretion into bile or urine. Preclinical and clinical data suggest that renal clearance of intact GalNAc siRNAs accounts for less than $25 \%$ of the total clearance (unpublished internal data). Given these attributes, it is highly unlikely that GalNAc siRNAs would serve as substrates or inhibitors/inducers of drug-metabolizing enzymes and transporters. Additionally, given the mechanism of uptake and the rapid distribution into the liver, the circulating plasma levels are low and transient. Furthermore, the free intracellular hepatocyte concentration is also likely to be low since ASGPR-mediated endocytosis deposits the siRNA into the lysosomal compartment (Weigel and Yik, 2002). A recent publication evaluated the potential for antisense oligonucleotides (ASOs) to mediate DDI based on in vitro investigations (Shemesh et al., 2017), but there are no published data on GalNAc siRNAs. As part of the nonclinical characterization, the DDI potential toward P450s and transporters was evaluated for multiple GalNAc siRNAs during their development. The assays conducted were dependent on the development stage for the GalNAc siRNA; for example, full transporter studies were only conducted on those in late stage (phase 2/3) development. These data indicate that the likelihood for clinical DDI at pharmacologically relevant doses is low.

\section{Materials and Methods}

\section{GalNAc siRNAs}

All oligonucleotides were synthesized as previously described by Nair et al. (2014). The general chemical modifications used in inclisiran, revusiran, fitusiran, and givosiran have been previously published (Shen and Corey, 2018). The designs of all other GalNAc-siRNA are consistent with those already published with or without the addition of a glycol nucleic acid (Schlegel et al., 2017).

Chemicals and Reagents. Antipyrine, 2-benzoxalinone, carbutamide, reserpine, warfarin, phenacetin, bupropion, paclitaxel, diclofenac, dextromethorphan, omeprazole, phenobarbital, rifampicin, estrone-3-sufate sodium salt, taurocholic acid sodium salt, cyclosporin A, metformin, pyrimethamine, verapamil hydrochloride, probenecid, $\beta$-estradiol 17-( $\beta$-D-glucuronide) sodium salt, cholecystokinine8, NADPH, DMSO, 1.0 M potassium phosphate monobasic solution, and 1.0 M potassium phosphate dibasic solution were purchased from Sigma Chemicals (St. Louis, MO). Midazolam, $S$-mephenytoin, $6 \alpha$-hydroxypaclitaxel, $6 \alpha$-hydroxypaclitaxel- $\mathrm{d}_{5}$, and $( \pm)$-4-hydroxymephenytoin- $\mathrm{d}_{3}$ were purchased from Toronto Research Chemicals (Toronto, Canada). Acetonitrile and methanol were purchased from Fisher Scientific (Pittsburgh, PA). Recombinant human P450s (rCYPs), ITS + Premix, and Matrigel were purchased from Corning (Tewksbury, MA). Pooled human liver microsomes (HLMs) (lot No. 1210223 , mixed gender, pool of 200 donors) were purchased from XenoTech, LLC (Lenexa, KS). Acetaminophen, hydroxybupropion, 4'-hydroxydiclofenac, 4'-hydroxymephenytoin, dextrorphan tartrate, $1^{\prime}$-hydroxymidazolam, $6 \beta$-hydroxytestosterone, acetaminophen $-\mathrm{d}_{4},( \pm)$-hydroxybupropion- $\mathrm{d}_{6}, 4^{\prime}$-hydroxydiclofenac- ${ }^{13} \mathrm{C}_{6}$, dextrorphan- $\mathrm{d}_{3}, 1^{\prime}$-hydroxymidazolam- $\mathrm{d}_{4}$, and $6 \beta$-hydroxytestosterone$d_{3}$ were purchased from Cerilliant (Round Rock, TX). The 96-well polypropylene assay plates and rubber sealing mats were purchased from Fisher Scientific. Ko134, (tetracyclic fumitremorgin $\mathrm{C}$ analog), ${ }^{3} \mathrm{H}-\mathrm{N}$-methyl-quinidine, $\mathrm{N}$-methylquinidine, and ${ }^{3} \mathrm{H}$-estrone-3-sulfate were supplied by Solvo (Szeged, Hungary). ${ }^{3} \mathrm{H}$-taurocholic acid, ${ }^{3} \mathrm{H}$-estradiol $17 \beta$-D glucuronide, and ${ }^{3} \mathrm{H}$-cholecystokinine- 8 were supplied by Perkin Elmer (Waltham, MA). ${ }^{3} \mathrm{H}$-tenofovir and ${ }^{14} \mathrm{C}$-metformin were supplied by Moravek Biochemicals (Brea, CA), and tenofovir was supplied by Sequoia Research Products Ltd.

\section{Determination of Incubation Concentrations for In Vitro Assays}

The concentrations of GalNAc siRNAs used in the in vitro assays were selected by considering the observed plasma concentrations and estimated liver concentrations in the clinic (Table 1). Since GalNAc siRNAs are large, anionic, and hydrophilic in nature, they do not passively cross plasma membranes. These siRNAs do not bind to albumin (data on file) and are unlikely to bind to liver microsomes or hepatocytes. A recent publication evaluated the potential for a different design of chemically modified GalNAc siRNAs to bind to plasma and liver proteins (Humphreys et al., 2019) in which the data indicated that binding to liver homogenate appeared greater than plasma binding. However, these studies were designed with a final protein concentration of $200 \mathrm{mg} / \mathrm{ml}$ (personal communication with first author). This protein concentration is much greater than what was used during the in vitro DDI investigations of $0.1-1 \mathrm{mg} / \mathrm{ml}$ contained herein. If protein binding is estimated from these reported data $\left(F_{\mathrm{u}, \text { liver }}=0.018-0.051\right)$, with the assumption that binding to liver homogenate

TABLE 1

Clinical pharmacokinetic parameters

\begin{tabular}{|c|c|c|c|}
\hline \multirow{2}{*}{ GalNAc siRNA } & \multirow{2}{*}{ Clinical Dose $^{a}$} & \multicolumn{2}{|c|}{ Clinical $C_{\max }$} \\
\hline & & Plasma & Liver Projected \\
\hline & & $\mu M$ & $\mu M$ \\
\hline Revusiran & $500 \mathrm{mg}$ & 0.058 & 5.1 \\
\hline HBV01 & $3(\mathrm{mg} / \mathrm{kg}$, highest dose tested $)$ & 0.057 & 12 \\
\hline AAT01 & $6(\mathrm{mg} / \mathrm{kg}$, highest dose tested $)$ & 0.11 & 29 \\
\hline Fitusiran & $80 \mathrm{mg}$ & 0.0092 & 4.3 \\
\hline Givosiran & $2.5(\mathrm{mg} / \mathrm{kg})$ & 0.020 & 12 \\
\hline Inclisiran & $300 \mathrm{mg}$ & 0.061 & 17 \\
\hline Cemdisiran & $600 \mathrm{mg}$ & 0.048 & 12 \\
\hline Vutrisiran & $25 \mathrm{mg}$ & 0.0054 & 1.9 \\
\hline Lumasiran & $3(\mathrm{mg} / \mathrm{kg})$ & 0.061 & 7.1 \\
\hline
\end{tabular}

${ }^{a}$ Clinically relevant unless otherwise noted. 
is linear to the protein level, than binding under the assay conditions employed $(0.1$ or $1 \mathrm{mg} / \mathrm{ml})$ is expected to be negligible $\left(F_{\mathrm{u}, \text { liver }}=0.8-1\right)$. This approach has previously been published (Kalvass et al., 2007; Sane et al., 2016; Riccardi et al., 2017). Furthermore, chemically modified GalNAc siRNAs are highly stable in liver in vivo and are not expected to be appreciably metabolized during the short incubation times employed in the in vitro studies (Nair et al., 2017). Therefore, no corrections are made for metabolic stability and plasma protein binding (assumed to be 1 ), and the $K_{\mathrm{i}}$ values estimated from $\mathrm{IC}_{50}$ values are expected to be representative of free values. It should be noted that the use of $F_{\mathrm{u}}=1$ is highly conservative since the plasma protein binding at relevant clinical concentrations is $\sim 90 \%\left(F_{\mathrm{u}}=0.1\right)$.

\section{P450 Substrate Study}

Recombinant enzymes were thawed rapidly at $37^{\circ} \mathrm{C}$ and kept on ice until use per the manufacturer's recommendation. Individual rCYP incubations contained $50 \mathrm{pmol} / \mathrm{ml}$ of rCYP1A2, rCYP2B6, rCYP2C8, rCYP2C9, rCYP2C19, rCYP2D6, rCYP3A4, or rCYP3A5, two concentrations of GalNAc siRNA, or $1 \mu \mathrm{M}$ of $\mathrm{P} 450$ probe substrate. Reactions were initiated by the addition of NADPH (1 mM final concentration). Protein concentrations for all enzymes were normalized to the recombinant $\mathrm{P} 450$ containing the highest protein concentration (Supplemental Table 1). All reactions were carried out in triplicate and the final organic solvent concentration in the incubation was $\leq 1 \%$. Reactions containing test article were terminated at 0 and 30 minutes by freezing on contact with dry ice and ethanol. Reactions containing positive controls were terminated by adding $100 \mu \mathrm{l}$ aliquot of the reaction mixture to $100 \mu \mathrm{l}$ of ice-cold acetonitrile (ACN) containing a cocktail of internal standard $(0.5 \mu \mathrm{M}$ of reserpine, antipyrine, carbutamide, warfarin, and $1 \mu \mathrm{M}$ 2-benzoxazolinone). Probe substrates for each of the rCYP enzymes were included in the study as positive controls. The negative control incubations consisted of the test article with rCYPs but without NADPH. GalNAc siRNA was analyzed by liquid chromatography (LC) time-of-flight (TOF) mass spectrometry (MS). Positive control substrates were analyzed by ultra-high-performance liquid chromatography-tandem mass spectrometry (LCMS/MS). Peak area ratios were used to calculate the percentage of parent remaining following 30- or 45-minute incubation.

\section{P450 Direct and Time-Dependent Inhibition Study}

The substrate concentrations used were selected to approximate the $K_{\mathrm{m}}$ values determined for each batch of HLMs used. Potassium phosphate buffer (100 mM, $\mathrm{pH}$ 7.4) was prepared by first diluting $1 \mathrm{M}$ solutions of monobasic and dibasic potassium phosphate with ultrapure water to $0.1 \mathrm{M}$. The $100 \mathrm{mM}$ solutions were combined in a $\sim 3: 1$ ratio of dibasic:monobasic potassium phosphate such that the final $\mathrm{pH}$ of the buffer was 7.4. HLMs at $20 \mathrm{mg} / \mathrm{ml}$ were diluted in the reaction mixture to $0.1 \mathrm{mg} / \mathrm{ml}$ for incubation with the probe substrate. The probe substrate assay conditions were designed by considering the linearity of the reaction and ensuring that minimal $(<10 \%)$ depletion of substrates occurred over the incubation time course (characterization data on file at the contract research organization). Reactions were started by the addition of $20 \mu \mathrm{l}$ of $10 \mathrm{mM}$ NADPH $(10 \times)$ and returned to the incubated shaker. The total incubation volume was $200 \mu$ l. All incubations were conducted at $37^{\circ} \mathrm{C}$. Reactions proceeded for 5 minutes (midazolam), 10 minutes (phenacetin, dextromethorphan, and testosterone), 15 minutes (bupropion, diclofenac), 25 minutes ( $S$-mephenytoin), or 40 minutes (paclitaxel). At the end of the incubation, $100 \mu \mathrm{l}$ of each reaction was terminated with an equal volume of ice-cold ACN containing a cocktail of stable, labeled internal standards. Time-dependent inhibition of P450 activities was performed using pooled HLMs with 30-minute preincubation. Pooled HLMs $(1 \mathrm{mg} / \mathrm{ml}$ ) were preincubated for 30 minutes with GalNAc siRNA (10 concentrations in triplicate) in potassium phosphate buffer in the presence or absence of $1 \mathrm{mM}$ NADPH at $37^{\circ} \mathrm{C}$. Reactions $(50 \mu \mathrm{l})$ containing potassium phosphate, HLMs, and NADPH were started with $12.5 \mu \mathrm{l}$ of the $4 \times$ inhibitor dilution, blank, or $4 \times$ positive control inhibitor. Mechanism-based selective inhibitors furafylline (1 $\mu \mathrm{M}$ for CYP1A2), ThioTEPA (5 $\mu \mathrm{M}$ for CYP2B6), gemfibrozil glucuronide (25 $\mu \mathrm{M}$ for CYP2C8), tienilic acid ( $1 \mu \mathrm{M}$ for CYP2C9), ticlopidine (10 $\mu \mathrm{M}$ for CYP2C19), paroxetine (0.5 $\mu \mathrm{M}$ for CYP2D6), and azamulin (0.5 $\mu \mathrm{M}$ for CYP3A4/5) were included as the positive controls in triplicate. Following the 30-minute preincubation time period a $20 \mu \mathrm{l}$ aliquot was taken from each well and added to $180 \mu \mathrm{l}$ of reaction mix. NADPH was approximately $1 \mathrm{mM}$ and the HLMs were $0.1 \mathrm{mg} / \mathrm{ml}$. The plates containing the substrate reactions were placed on the incubation shaker and incubated for 5 minutes (midazolam), 10 minutes (phenacetin, dextromethorphan, and testosterone), 15 minutes (bupropion and diclofenac), 25 minutes ( $S$-mephenytoin), or 40 minutes (paclitaxel). At the end of the incubation, reactions were terminated by mixing $100 \mu \mathrm{l}$ of each reaction mixture with an equal volume of ice-cold ACN containing a cocktail of stable, labeled internal standards.

\section{P450 Induction Study}

Cryopreserved human hepatocytes from three different human donors were used for evaluation of induction potential. The donor demographics are given in Supplemental Table 2. Frozen vials of cryopreserved hepatocytes were rapidly thawed in a $37^{\circ} \mathrm{C}$ water bath and resuspended in hepatocyte plating medium containing $\sim 30 \%$ Percoll. The cells were centrifuged and resuspended in hepatocyte plating medium and the viability and cell count were determined using the Nexcelom Cellometer according to the manufacturer's procedures. Cells were only used if the final isolation viability was greater than $80 \%$. Hepatocyte plating medium was added to bring the cells to the required concentration and seeded onto 24-well plates coated with a simple collagen, type I substratum. Cells were incubated in a humidified chamber at $37^{\circ} \mathrm{C}$ with $5 \% \quad \mathrm{CO}_{2}$ and cell attachment was confirmed 4-6 hours after plating by visual inspection using phase-contrast microscopy. After attachment, plates were swirled, and medium containing debris and unattached cells was aspirated. Fresh hepatocyte maintenance media (HMM) containing ITS + Premix and Matrigel was added to culture plates and the plates were immediately returned to the humidified incubation chamber. Hepatocyte cultures were maintained for approximately 24 hours prior to treatment with GalNAc siRNAs or prototypical inducers. Positive and negative controls were prepared at $1000 \times$ the final concentration in DMSO. Working solutions were prepared by dilution of the $1000 \times$ solutions in HMM $(1 \mu$ l stock + $999 \mu 1$ media). The final concentration of DMSO under all conditions was $0.1 \%$. Cultures were treated for two or three consecutive days for reverse transcription (RT) polymerase chain reaction or in situ activity, respectively, with solvent alone (0.1\% DMSO), positive controls (50 $\mu \mathrm{M}$ omeprazole, $1 \mathrm{mM}$ phenobarbital, and $10 \mu \mathrm{M}$ rifampicin), negative control (25 $\mu \mathrm{M}$ flumazenil), or test article. All treatments were carried out in triplicate. The medium was replaced daily with fresh supplemented medium containing the appropriate treatment. Prior to mRNA preparation at 48 hours of treatment or in situ P450 activity determination at 72 hours, $100 \mu \mathrm{l} /$ well of culture supernatant was taken and assayed for lactate dehydrogenase leakage using the CytoTox-ONE Homogeneous Membrane Integrity Assay Kit (Promega, Madison, WI). After 48 hours of treatment, spent medium from a subset of each treatment group was aspirated and cells were lysed for mRNA preparation with $200 \mu \mathrm{l}$ of 1:1 Buffer RLT (Qiagen) and Trizol (Life Technologies, Carlsbad, CA). RNA was isolated using the RNeasy Kit (Qiagen) according to the manufacturer's instructions. Eluted RNA was quantified using a NanoDrop spectrophotometer (ThermoFisher, Pittsburgh, PA). RT was performed with the High Capacity cDNA Reverse Transcriptase Kit with RNase inhibitor (Life Technologies) using an ABI 9700 thermocycler. Quantitative polymerase chain reaction analysis was performed on RT reactions using gene-specific primer/probe sets for CYP1A2, CYP2B6, or CYP3A4 target cDNA and endogenous control (glyceraldehyde-3phosphate dehydrogenase). Relative fold change in mRNA content was determined based on the threshold cycle $\left(\mathrm{C}_{\mathrm{T}}\right)$ data of each target gene relative to endogenous control for each reaction and normalized to vehicle control. After 72 hours of treatment, the spent medium was aspirated from the remaining cultures from each treatment group, wells were washed with warmed HMM, and a cocktail of P450 probe substrates in HMM was added to each well (phenacetin, $100 \mu \mathrm{M}$; bupropion, $50 \mu \mathrm{M}$; and midazolam, $10 \mu \mathrm{M}$ ). Probe substrate incubations were performed for 30 minutes at $37^{\circ} \mathrm{C}$ on a rotary shaker at $150 \mathrm{rpm}$ and terminated by taking $100 \mu \mathrm{l}$ of the reaction and adding it to $100 \mu \mathrm{l} /$ well of ice-cold ACN with stable, labeled internal standards. Plates were vortexed for 4 minutes, centrifuged at $3000 \mathrm{rpm}$ for 10 minutes at $4{ }^{\circ} \mathrm{C}$, and then analyzed for quantitation of metabolite levels.

\section{Evaluation of GalNAc-siRNA Uptake into Sandwich Cultured Hepatocytes}

Cryopreserved human hepatocytes from a single donor were used to determine whether the human hepatocytes were exposed to GalNAc siRNAs evaluated in the P450 induction study. Human hepatocytes were recovered, plated, and cultured as described in P450 Induction Study. After the acclimation period, the same concentrations of GalNAc siRNAs used to evaluate induction potential were 
added to the hepatocytes. Twenty-four hours post GalNAc-siRNA administration the hepatocyte cultures were washed twice with ice-cold PBS and $100 \mu \mathrm{l}$ of lysis buffer containing $0.25 \%$ Triton X-100 in 1X PBS was added to lyse the cells. Cells were scraped from the bottom of the well and test article concentrations were determined. The concentration of test articles in the in vitro hepatocyte samples were determined using a RT quantitative polymerase chain reaction method capable of quantitating antisense strands of siRNA using a target-specific stem-loop primer and sequence-specific forward and reverse primers for amplification using the general conditions previously described (Landesman et al., 2010). PBS/Triton X-100 buffer was spiked with test articles to generate the standard curves and quality control samples. Concentrated samples were diluted in $0.25 \%$ PBS/Triton X-100. Intracellular concentrations were estimated as previously described (Ramsden et al., 2014), using the viable seeded cell number of 65,000 cells/well.

\section{In Vitro Transporter Substrate Study}

Specific transporter assay details are presented in Supplemental Table 3. The accumulation of GalNAc siRNAs into membrane vesicles was determined using inside-out membrane vesicles (total protein: $50 \mu \mathrm{g} / \mathrm{well}$ for all transporters) prepared from cells overexpressing human ATP-binding cassette transporters as well as from control cells. Two incubation time points ( 2 and 20 minutes) and two concentrations of GalNAc siRNAs were tested in the presence of ATP or AMP to determine whether the GalNAc siRNA is actively transported into the vesicles. Reactions were quenched by the addition of $200 \mu 1$ of ice-cold washing buffer and immediate filtration via glass fiber filters mounted to a 96-well plate (filter plate). The filters were washed five times with $200 \mu \mathrm{l}$ of ice-cold washing buffer and dried. GalNAc siRNA was extracted from the vesicles through lysing them two times with $100 \mu \mathrm{l}$ of lysis solution [Epicentre, Tissue \& Cell Lysis Solution $(600 \mathrm{ml})]$. Samples were analyzed to determine the concentration of GalNAc siRNA retained inside the vesicles determined by LC-MS/MS detection. Incubation with AMP provided background activity values for all data points. Incubations with probe substrate (solvent only) served as the positive controls, while membrane vesicle preparations from control cells [for breast cancer resistance protein (BCRP) and P-glycoprotein (P-gp)] or Sf9 cells expressing $\beta$-gal [for bile salt export pump (BSEP)] served as the negative controls.

\section{In Vitro Transporter Inhibition Study: Vesicular Assays}

GalNAc siRNA was incubated with membrane vesicle preparations (total protein: $25 \mu \mathrm{g} / \mathrm{well}$ for BCRP and $50 \mu \mathrm{g} / \mathrm{well}$ for BSEP and P-gp) and the probe substrate. Incubations were carried out in the presence of $4 \mathrm{mM}$ ATP or AMP to distinguish between transporter-mediated uptake and passive diffusion into the vesicles. GalNAc siRNA was added to the reaction mixture in $1.5 \mu \mathrm{l}$ of solvent. Reaction mixtures were preincubated for 10 minutes at $32 \pm 1^{\circ} \mathrm{C}$ for BCRP and at $37 \pm 1^{\circ} \mathrm{C}$ for BSEP and P-gp. Reactions were initiated by the addition of $25 \mu \mathrm{l}$ of $12 \mathrm{mM} \mathrm{MgATP}$ (or $12 \mathrm{mM}$ AMP in assay buffer as a background control) and preincubated separately. Reactions were quenched by the addition of $200 \mu \mathrm{l}$ of ice-cold washing buffer and immediate filtration via glass fiber filters mounted on a 96-well plate (filter plate). The filters were washed five times with $200 \mu$ l of icecold washing buffer, dried, and then the amount of substrate inside the filtered vesicles was determined by liquid scintillation counting.

\section{In Vitro Transporter Substrate Study: Uptake Assays}

Uptake experiments were performed using $\mathrm{CHO}$, Madin-Darby canine kidney II, or human embryonic kidney 293 cells stably expressing the respective uptake transporters. Cells were cultured at $37 \pm 1^{\circ} \mathrm{C}$ in an atmosphere of air: $\mathrm{CO}_{2}(95: 5)$ as described in Supplemental Table 3 and plated onto standard 24-well tissue culture plates at a density $5 \times 10^{5}$ cells/well. The uptake of GalNAc siRNA was determined using cells overexpressing the respective uptake transporters [multidrug and toxin extrusion protein (MATE) 1, MATE2-K, organic anion transporter (OAT) 1, OAT3, organic anion transporting polypeptide (OATP) 1B1, OATP1B3, organic cation transporter (OCT) 1, and OCT2] and control cells, at two incubation time points ( 2 and 20 minutes) and two concentrations of GalNAc siRNAs. In follow-up inhibition assays conducted for MATE1, OATP1B1, and OAT3, the transporter-specific uptake of the GalNAc siRNAs was determined in the presence of a known inhibitor of the respective transporter $(1 \mu \mathrm{M}$ pyrimethamine for MATE1, $50 \mu \mathrm{M}$ rifampicin for OATP1B1, and $200 \mu \mathrm{M}$ probenecid for OAT3).

Before the experiment, the medium was removed, and the cells were washed twice with $300 \mu \mathrm{l}$ of $\mathrm{HK}$ buffer (pH 7.4 or 8.0). Cellular uptake of GalNAc siRNA into the cells was measured by adding $300 \mu \mathrm{l}$ of HK buffer containing GalNAc siRNA and incubating them at $37 \pm 1{ }^{\circ} \mathrm{C}$. Reactions were quenched by removing the HK buffer containing GalNAc siRNA and the cells were washed twice with $300 \mu l$ of HK buffer. Cells were lysed by adding $300 \mu \mathrm{l}$ of lysis solution [Epicentre, Tissue \& Cell Lysis Solution $(600 \mathrm{ml})]$ and incubated for 10 minutes. Samples were analyzed to determine the concentration of GalNAc siRNA retained inside the vesicles determined by LC-MS/MS detection. The amount of protein in each well was quantified using the BCA Kit for protein determination (Sigma-Aldrich).

\section{In Vitro Transporter Inhibition Study: Uptake Assays}

Cells were cultured at $37 \pm 1{ }^{\circ} \mathrm{C}$ in an atmosphere of air: $\mathrm{CO}_{2}$ (95:5) and plated onto standard 96-well tissue culture plates at the cell number described in Supplemental Table 3. Before the experiment, the medium was removed and the cells were washed twice with $100 \mu 1$ of HK buffer at pH 7.4 (OAT1, OAT3, OATP1B1, OATP1B3, OCT1, and OCT2) or 8.0 (MATE1 and MATE2-K). Uptake experiments were carried out at $37 \pm 1^{\circ} \mathrm{C}$ in $50 \mu$ of $\mathrm{HK}$ buffer ( $\mathrm{pH} 7.4$ or 8.0) containing the probe substrate and GalNAc siRNA or solvent. The organic solvent concentration was equal in all wells. After the experiment, cells were washed twice with $100 \mu \mathrm{l}$ of ice-cold HK buffer and lysed with $50 \mu$ lof $0.1 \mathrm{M} \mathrm{NaOH}$. Radiolabeled probe substrate transport was determined by measuring an aliquot $(35 \mu \mathrm{l})$ from each well for liquid scintillation counting. Uptake of the probe substrate in control cells provided background activity values for all data points. Incubation without GalNAc siRNA or reference inhibitor (PBS only) provided 100\% activity values. A reference inhibitor served as the positive control for inhibition.

Analytical Methods. Samples $(20 \mu 1)$ were injected on a Supelco Discovery $\mathrm{C} 185 \mu 2.1 \times 150 \mathrm{~mm}$ column maintained at room temperature using a Thermo Accela High Speed LC system. A solvent system consisting of water with $0.1 \%$ formic acid (mobile phase A) and ACN with $0.1 \%$ formic acid (mobile phase B) at a flow rate of $0.4 \mathrm{ml} / \mathrm{min}$ was used with the following gradient: $0-5$ minutes, $15 \%$ B; 5-10 minutes, $15 \%-52.5 \%$ B; 15 to 16 minutes, 52.5\%-85\% B and maintain at $85 \%$ B for 2 minutes; 18 to 19 minutes, $15 \% \mathrm{~B}$; and then equilibrated for 3 minutes. The column eluent was analyzed by MS on a Thermo LTQ OrbiTrap XL Mass Spectrometer run in positive mode using Fourier transform MS and data-dependent Fourier transform MS/MS scans (collision-induced dissociation and higher-energy collisional dissociation) to elucidate metabolite structures. The resulting data were analyzed using Xcalibur 3.0 and Thermo Metworks 1.3 software.

\section{Analysis of GalNAc-siRNA Concentrations}

LC-TOF-MS assays were developed. The LC-TOF-MS assays quantified the concentrations of specific GalNAc siRNAs by the peak area ratio and by detecting the antisense strand and sense strand portions of the duplex (by the peak area ratio only). Incubation samples were spiked with internal standard (IS), processed by solidphase extraction, and analyzed using reversed-phase ultra-high-performance LC with Turbo Ion Spray TOF-MS detection. Accurate masses of 10 ions for each strand of the analyte, sense, and antisense and each strand of the IS, antisense, and sense were monitored in the negative ion mode. The peak area for the analyte or IS was the sum of the response from the respective 10 ions. The peak area ratios of the respective analyte-sense/IS-sense and analyte-antisense/IS-antisense single strands were used.

\section{Probe Substrate P450 Assays}

The quenched reactions were vortexed at high speed (knob setting 10) for 4 minutes and centrifuged for 10 minutes at $3000 \pm 40 \mathrm{rpm}$ at low temperature $\left(\sim 10^{\circ} \mathrm{C}\right)$. A portion of the supernatant $(150 \mu \mathrm{l})$ was transferred into $1.4 \mathrm{ml}$ snaplock tubes (Nova Biostorage). Samples were centrifuged for 5 minutes as described previously prior to injection on the ultra-high-performance LC-MS/MS. The analysis system consisted of a Shimadzu Nexera $\times 2$ LC-30 UPLC system (Shimadzu Corporation, Kyoto, Japan) and an API4000 triple quadrupole mass spectrometer (AB Sciex, Toronto, Canada). The LC-MS/MS system was controlled by Analyst software (version 1.6.1). Analyst performed the system hardware control and sample analysis. The following transitions were used in the probe substrate assays: phenacetin $(180.12 \rightarrow 138.17)$, acetaminophen $(152.1 \rightarrow$ 110.1), bupropion ( $240.11 \rightarrow 184.07)$, ( \pm )-hydroxybupropion $(256.1 \rightarrow 131.2)$, paclitaxel $(854.46 \rightarrow 569.20)$, diclofenac $(296.32 \rightarrow 214.07), S$-mephenytoin $(219.19 \rightarrow 134.09)$, dextromethorphan $(272.26 \rightarrow 215.15)$, midazolam $(326.14 \rightarrow$ 291.12), 1'-hydroxymidazolam $(342.1 \rightarrow 203.1)$, internal standard warfarin $(309.39 \rightarrow 163.00)$, acetaminophen- $\mathrm{d}_{4}(156.1 \rightarrow 114.1), 1^{\prime}$ '-hydroxymidazolam$\mathrm{d}_{4}(346.1 \rightarrow 03.1)$, and hydroxybupropion- $\mathrm{d}_{6}(262.1 \rightarrow 131.2)$. 


\section{Calculations and Data Analysis}

IC $_{50}$ Values. The concentrations of the metabolites formed were calculated from a calibration curve using authentic metabolite standards. The $\mathrm{P} 450$ activities were expressed as picomoles per minute per milligram of microsomes. The degree of inhibition was determined by comparing the formation of metabolites in the presence of different concentrations of GalNAc siRNA versus vehicle control. The $\mathrm{IC}_{50}$ values were calculated from the percentage of vehicle control activity using GraphPad Prism 7 for Windows (GraphPad Software, La Jolla, CA) and applying eq. 1. Comparison of three parameter (hillslope $=-1$ ) or four parameter fitting was conducted to determine the best fit. Curve fitting was not carried out in cases where the inhibition at the highest concentration of test article was $\leq 10 \%$ of the control value, rather the $\mathrm{IC}_{50}$ value was set to greater than the highest concentration of GalNAc siRNA tested. The calculated $\mathrm{IC}_{50}$ values for positive controls were compared with reported literature values and results typically obtained at QPS (Newark, DE)

$$
Y=\min +\frac{\max -\min }{1+\left(x / \mathrm{IC}_{50}\right)^{- \text {hillsslope }}}
$$

To determine if the test article is a time-dependent inhibitor, changes in the $\mathrm{IC}_{50}$ value from preincubated samples with $\mathrm{NADPH}$ were compared with the $\mathrm{IC}_{50}$ value from samples preincubated without NADPH. Time-dependent inhibition was indicated if the ratio of $\mathrm{IC}_{50}$ (without $\mathrm{NADPH} / \mathrm{IC}_{50}$ (with $\mathrm{NADPH}$ ) was $>1.5$ (Grimm et al., 2009). For the assay to be considered valid, the timedependent controls had to cause at least a $20 \%$ change in the percentage of inhibition in the presence of NADPH.

The $K_{\mathrm{i}}$ values were estimated from the $\mathrm{IC}_{50}$ values using the Cheng-Prusoff equation (Cheng and Prusoff, 1973), assuming a competitive inhibition mechanism:

$$
\mathrm{IC}_{50}=K_{\mathrm{i}} \cdot\left(1+\frac{[S]}{K_{\mathrm{m}}}\right)
$$

Induction Data. TaqMan data were analyzed using the comparative $\mathrm{C}_{\mathrm{T}}$ method (Schmittgen and Livak, 2008) (also known as the $\Delta \Delta \mathrm{C}_{\mathrm{T}}$ method). The $\mathrm{C}_{\mathrm{T}}$ values from the samples and the glyceraldehyde-3-phosphate dehydrogenase controls were used for the calculations. The $\Delta \mathrm{C}_{\mathrm{T}}$ was calculated by subtracting the $\mathrm{C}_{\mathrm{T}}$ value of the reference (glyceraldehyde-3-phosphate dehydrogenase) from the $\mathrm{C}_{\mathrm{T}}$ value of the sample. Next, the $\Delta \Delta \mathrm{C}_{\mathrm{T}}$ value was calculated by subtracting the mean $\Delta \mathrm{C}_{\mathrm{T}}$ of the calibrator (the vehicle control) from each sample $\Delta \mathrm{C}_{\mathrm{T}}$. The relative quantitation (or fold over control) was calculated by raising 2 to the negative power of the $\Delta \Delta \mathrm{C}_{\mathrm{T}}$.

Statistical Analyses. The mean values of all replicates were used in the calculations. The $\mathrm{IC}_{50}$ values were determined using GraphPad Prism (version 7). Data were processed with Microsoft Excel 2010 (Microsoft, Redmond, WA). To evaluate significance for induction studies, data were analyzed using one-way ANOVA with the results examined for statistical significance using the Bonferroni procedure in the XLSTAT add-in for Microsoft Excel (Addinsoft, New York, NY). When necessary, data were examined for outliers using the Grubbs' test (Grubbs, 1950). The Grubbs' Test for Outliers module of the XLSTAT add-in for Microsoft Excel (Addinsoft, New York, NY) was used to perform the analysis. All studies were conducted at QPS, Solvo, or Sekisui XenoTech (Kansas City, KS) in accordance with company-specific standard operating procedures, in the spirit of good laboratory practice (GLP).

\section{Estimation of Liver Concentrations in Human}

The maximal concentration in human liver was estimated using Phoenix WinNonlin and two approaches. The first considered the relationship between rat and monkey liver to plasma ratios, where the monkey liver to plasma ratio was scaled allometrically to human based on body weight with an exponent of 0.37 . The exponent was derived using the average mouse, rat, and monkey liver to plasma ratios determined at nonsaturating dose levels of nine GalNAc siRNAs during pharmacokinetic/pharmacodynamic studies. The second approach relied on simulations derived from fitting of the average liver pharmacokinetic profile observed in monkey and allometric scaling clearance (CL) (exponent 0.75 ) and volume (V) (exponent 1). The estimated liver concentrations were within 2.5 -fold of each other using all methods and the mean concentration was used as an input for the DDI risk assessment. The estimated liver concentrations are based on total concentrations and not reflective of free concentrations or discriminative of location (i.e., hepatocytes, endocytic pathway vs. endoplasmic reticulum).

\section{Mechanistic Static Modeling}

The mechanistic static model reported previously (Fahmi et al., 2009) was used with the following exceptions. Equation 2 was reduced to eq. 3 by considering the enzymes that were inhibited, the mode of GalNAc-siRNA delivery (subcutaneous rather than oral), and the interactions observed (direct inhibition only):

$$
\begin{aligned}
\mathrm{AUCi} / \mathrm{AUC}= & \left(\frac{1}{\left[A_{h} \times B_{h} \times C_{h}\right] \times f_{m}+\left(1-f_{m}\right)}\right) \\
& \times\left(\frac{1}{\left[A_{g} \times B_{g} \times C_{g}\right] \times\left(1-f_{g}\right)+f_{g}}\right) \\
\mathrm{AUCi} / \mathrm{AUC}= & \left(\frac{1}{\left[A_{h}\right] \times f_{m}+\left(1-f_{m}\right)}\right)
\end{aligned}
$$

where

$$
A_{h}=\frac{1}{1+\left([I]_{h} / K_{\mathrm{i}}\right)}
$$

where AUC is the area under the concentration-time curve; AUCi is the area under the curve in the presence of the interactant; and $I=$ estimated liver concentration (Table 1).

\section{Results}

\section{P450 Substrate}

The potential of GalNAc siRNAs to act as a substrate of major P450 isoforms was evaluated using cDNA expressed enzymes (see Table 2). In vitro incubations were conducted in triplicate with two concentrations, each of seven GalNAc siRNAs that contain different oligonucleotide sequences and chemical modifications. No NADPH dependence and no meaningful depletion of the GalNAc siRNAs were observed after incubation with recombinant enzymes (Table 2). These studies were designed to evaluate both the sense and antisense strands. The positive

TABLE 2

CYP450 reaction phenotyping using $\mathrm{rCYP}$ and determining the \% siRNA remaining after designated time-course

\begin{tabular}{lccccccc}
\hline Parameter & Revusiran & HBV01 & AAT01 & Fitusiran & Cemdisiran & Vutrisiran & Lumasiran \\
\hline Time point $(\mathrm{min})$ & 45 & 45 & 45 & 45 & 45 & 45 & 45 \\
Concentration $(\mu \mathrm{M})$ & 0.620 & 0.053 & 0.614 & 0.0465 & 0.0477 & 0.0490 & 0.612 \\
CYP1A2 & 105 & 97.8 & 102 & 106 & 103 & 107 & 103 \\
CYP2B6 & 104 & 113 & 101 & 103 & 109 & 104 & 100 \\
CYP2C8 & 98.2 & 102 & 103 & 100 & 96.1 & 107 & 106 \\
CYP2C9 & 102 & 87.6 & 97.5 & 91.6 & 101 & 96.9 & 99.4 \\
CYP2C19 & 100 & 95.8 & 107 & 102 & 102 & 106 & 98.8 \\
CYP2D6 & 98.2 & 88.8 & 103 & 102 & 110 & 105 & 107 \\
CYP3A4 & 102 & 108 & 101 & 102 & 95.4 & 102 & 94.2 \\
CYP3A5 & 89.3 & 97.2 & 98.6 & 100 & 92.9 & 102 & 102 \\
\hline
\end{tabular}


control substrates all resulted in time-dependent depletion over the incubation time course, which was consistent with historical data, confirming that rCYPs were appropriately functional (Supplemental Table 4). Based on the minimal depletion observed, GalNAc siRNAs are not substrates of CYP1A2, 2B6, 2C8, 2C9, 2C19, 2D6, 3A4, or 3A5; therefore, clinical interactions with inhibitors and inducers of $\mathrm{P} 450$ enzymes are not likely.

\section{P450 Direct and Time-Dependent Inhibition}

The potential of 12 GalNAc siRNAs to inhibit the major $\mathrm{P} 450$ isoforms (i.e., CYP1A2, CYP2B6, CYP2C8, CYP2C9, CYP2C19, CYP2D6, and CYP3A) was evaluated in vitro using pooled HLMs and standard probe substrates for each $\mathrm{P} 450$ isoform, and included appropriate positive and negative controls. No direct or timedependent inhibition was observed for CYP1A2, 2C9, 2C19, 2D6, or 3A4/5 by any of the GalNAc siRNAs evaluated (Fig. 1; Tables 3,
4, and 5). AAT02 was also evaluated for time-dependent inhibition and resulted in an $\mathrm{IC}_{50}$ value $>18.4 \mu \mathrm{M}$ with and without NADPH for the same P450s (data not included in Tables 3, 4, or 5). Additionally, although not requested to be evaluated by regulatory authorities, fitusiran did not result in direct inhibition of CYP2A6. All prototypical direct and time-dependent inhibitors resulted in values in line with the literature reports (Supplemental Tables 5 and $6)$, demonstrating that the test system was functional for assessing inhibition potential. Cemdisiran, fitusiran, and lumasiran resulted in direct inhibition of CYP2C8 at very high incubation concentrations with $\mathrm{IC}_{50}$ values of 224,56 , and $416 \mu \mathrm{M}$, respectively. These values are $>4600$-fold higher than the total maximal steady-state concentrations observed in plasma. Cemdisiran also caused concentrationdependent direct inhibition of CYP2B6 with an $\mathrm{IC}_{50}$ value of $583 \mu \mathrm{M}$, which is 12,000 -fold higher than the therapeutically relevant total plasma $C_{\max }$ at steady state. No time-dependent inhibition was observed for CYP2C8 or CYP2B6 by any of the GalNAc
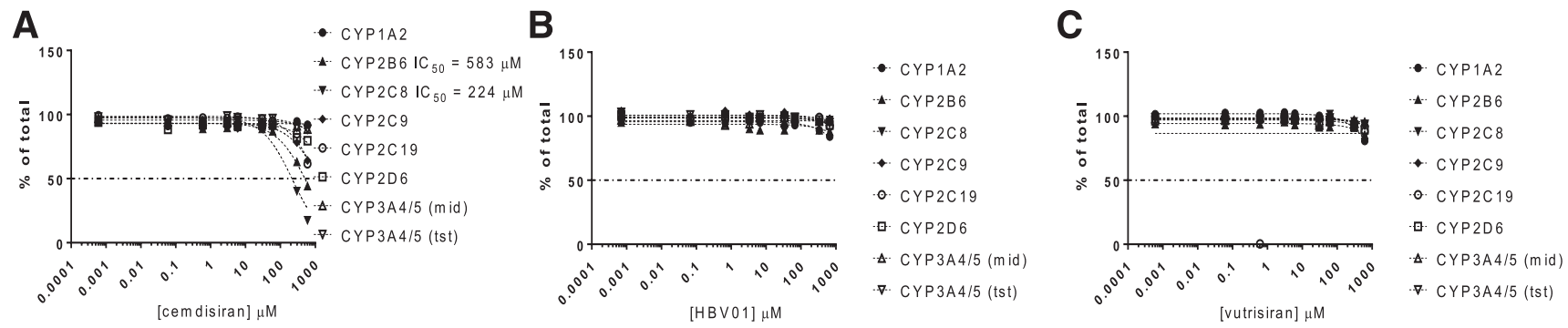

D

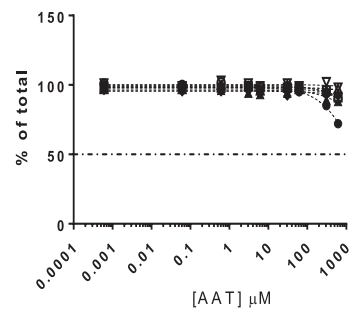

G

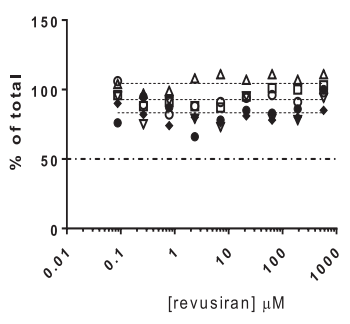

J

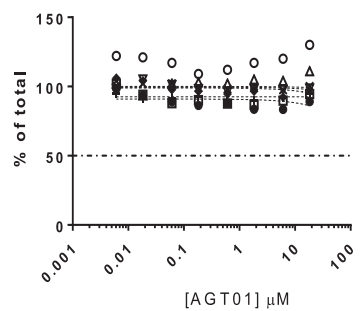

- CYP1A2

- CYP2B 6

-. $C Y P 2 C 8$

- $\mathrm{CYP} 2 \mathrm{C} 9$

- $C Y P 2 C 19$

- $C Y P 2 D 6$

A. CYP3A4/5 (mid)

7. CYP $3 A 4 / 5$ (tst)

E

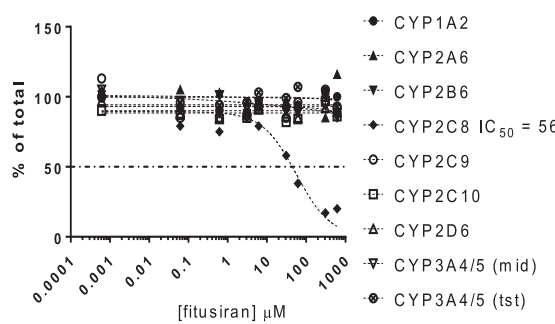

H
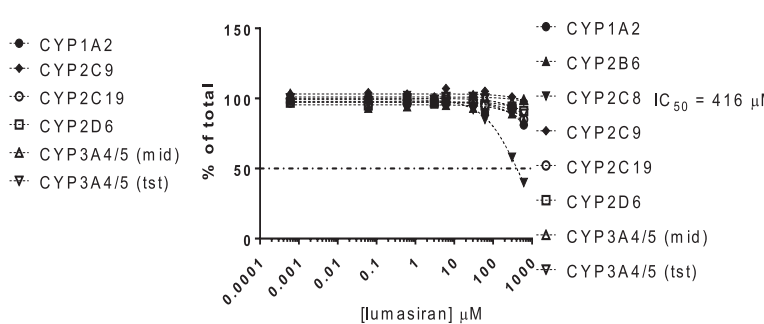

K

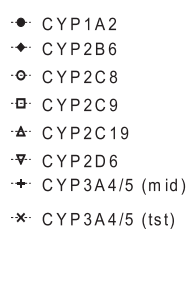

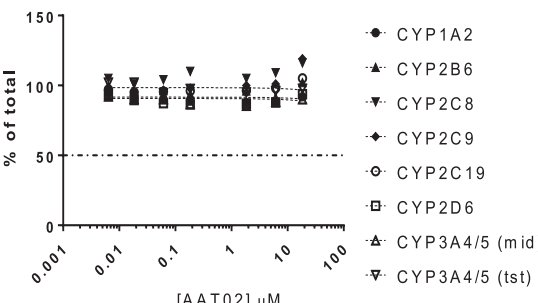

F

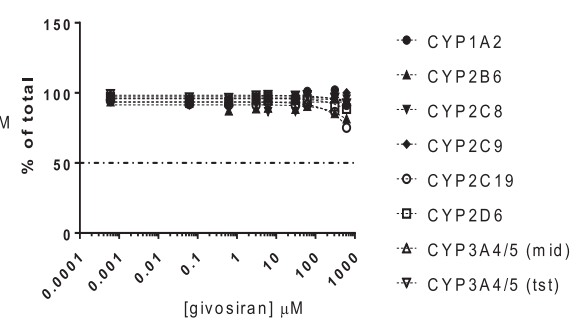

I

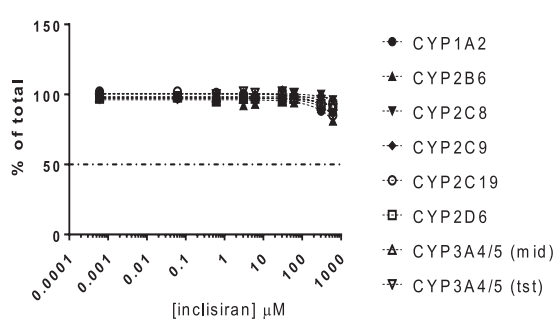

L

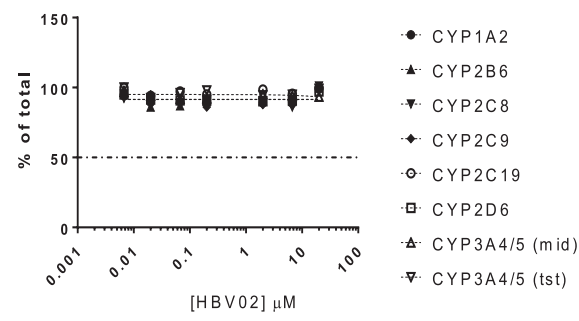

Fig. 1. The potential for cemdisiran (A), HBV01 (B), vutrisiran (C), AAT (D), fitusiran (E), givosiran (F), revusiran (G), lumasiran (H), inclisiran (I), AGT01 (J), AAT02 (K), or HBV02 (L) to inhibit the seven major P450 enzymes was investigated using pooled HLMs. No inhibition of CYP1A2, 2B6, 2C8, 2C9, 2C19, 2D6, and 3A4/5 was observed after incubation of HBV01, vutrisiran, AAT, givosiran, revusiran, inclisiran, AGT01, AAT02, or HBV02. Weak in vitro inhibition was observed for CYP2B6 and CYP2C8 by cemdisiran. Weak in vitro inhibition was observed for CYP2C8 after incubation with fitusiran and lumasiran. 
TABLE 3

Time-dependent inhibition results for GalNAc siRNAs against P450s

\begin{tabular}{|c|c|c|c|c|c|c|c|c|c|}
\hline \multirow{3}{*}{ P450 Isoform } & \multicolumn{9}{|c|}{$\mathrm{IC}_{50}(\mu \mathrm{M})$} \\
\hline & \multicolumn{3}{|c|}{ AAT01 } & \multicolumn{3}{|c|}{ HBV01 } & \multicolumn{3}{|c|}{ Givosiran } \\
\hline & $-\mathrm{NADPH}$ & $+\mathrm{NADPH}$ & Ratio & -NADPH & $+\mathrm{NADPH}$ & Ratio & -NADPH & $+\mathrm{NADPH}$ & Ratio \\
\hline CYP1A2 & $>614$ & $>614$ & 1 & $>665$ & $>665$ & 1 & $>614$ & $>614$ & 1 \\
\hline CYP2B6 & $>614$ & $>614$ & 1 & $>665$ & $>665$ & 1 & $>614$ & $>614$ & 1 \\
\hline CYP2C8 & $>614$ & $>614$ & 1 & $>665$ & $>665$ & 1 & $>614$ & $>614$ & 1 \\
\hline CYP2C9 & $>614$ & $>614$ & 1 & $>665$ & $>665$ & 1 & $>614$ & $>614$ & 1 \\
\hline CYP2C19 & $>614$ & $>614$ & 1 & $>665$ & $>665$ & 1 & $>614$ & $>614$ & 1 \\
\hline CYP2D6 & $>614$ & $>614$ & 1 & $>665$ & $>665$ & 1 & $>614$ & $>614$ & 1 \\
\hline CYP3A4/5 (midazolam) & $>614$ & $>614$ & 1 & $>665$ & $>665$ & 1 & $>614$ & $>614$ & 1 \\
\hline CYP3A4/5 (testosterone) & $>614$ & $>614$ & 1 & $>665$ & $>665$ & 1 & $>614$ & $>614$ & 1 \\
\hline
\end{tabular}

siRNAs tested. Considering the in vitro data and the recommendations in regulatory guidance documents (European Medicines Agency, 2012; Food and Drug Administration, 2017), the risk of clinical DDI due to inhibition is low.

\section{P450 Induction}

No concentration-dependent or statistically significant increase in mRNA levels was observed for any of the GalNAc siRNAs evaluated, while the positive and negative control compounds elicited expected magnitudes of response (Fig. 2). There were also no measurable increases in enzyme activity following any of the GalNAc-siRNA treatments (data on file). Metabolic stability for inclisiran was determined during the induction study and the results demonstrated that inclisiran was highly stable ( $<5 \%$ depletion) over the 24-hour exposure period (Table 6).

\section{Confirmation of Uptake into Sandwich-Cultured Hepatocytes}

Higher concentrations of GalNAc siRNAs were observed in hepatocytes than the nominal incubation concentrations, particularly at lower concentrations (Table 7). This is consistent with the knowledge that ASGPR mediates active uptake of GalNAc into hepatocytes. The saturation of ASGPR uptake is not unexpected at these concentrations and has been previously described (Bon et al., 2017). Taken together, these data confirm that lack of induction during the in vitro induction study is not due to lack of exposure to test article.

\section{Transporter Substrate}

Four GalNAc siRNAs were evaluated as potential substrates of uptake (OATP1B1, OATP1B3, OAT1, OAT3, OCT1, and OCT2) and efflux (BCRP, BSEP, MATE1, MATE2-K, and P-gp) transporters. No significant accumulation $>2$-fold or inhibition of uptake by selective inhibitors $>50 \%$ was observed for any of the GalNAc siRNAs tested against any of the transporters evaluated. One potential exception was fitusiran, which was flagged as a potential substrate of MATE2-K during an initial investigation based on the accumulation ratio ( $>2$-fold). A follow-up study showed an accumulation ratio for MATE2-K as $<2$-fold, but mild inhibition when a prototypical MATE2-K inhibitor was used. Given the variability observed between studies, the knowledge that substrates for MATE2-K are typically cations and are generally also transported by OCT2 (Yonezawa and Inui, 2011), and that fitusiran was not a substrate for OCT2, the in vitro data were deemed inconclusive. Furthermore, considering that the fraction of fitusiran excreted in urine from hemophilia patients after three monthly doses was $17 \%$ of the total administered drug and no active secretion was observed, this in vitro observation is likely not clinically relevant. Based on the totality of data, the likelihood for GalNAc siRNAs to act as substrates for transporters is low.

\section{Transporter Inhibition}

The potential for GalNAc siRNAs to inhibit uptake (OATP1B1, OATP1B3, OAT1, OAT3, OCT1, and OCT2) and efflux (BCRP, BSEP, MATE1, MATE2-K, and P-gp) transporters was assessed using transfected cell lines or vesicles expressing the transporter of interest. No significant inhibition was observed for uptake or efflux transporters, with the exception of concentration-dependent inhibition of P-gp by givosiran (Fig. 3). While a full concentration effect curve was not generated, an estimation of the $\mathrm{IC}_{50}$ value $(4.2 \mu \mathrm{M})$ was made for the purpose of conducting a clinical DDI risk assessment. This evaluation demonstrated that the in vitro inhibition of P-gp observed is not projected to be clinically relevant.

TABLE 4

Time-dependent inhibition results for GalNAc siRNAs against P450s

\begin{tabular}{|c|c|c|c|c|c|c|c|c|c|}
\hline \multirow{3}{*}{ P450 Isoform } & \multicolumn{9}{|c|}{$\mathrm{IC}_{50}(\mu \mathrm{M})$} \\
\hline & \multicolumn{3}{|c|}{ Lumasiran } & \multicolumn{3}{|c|}{ Cemdisiran } & \multicolumn{3}{|c|}{ Vutrisiran } \\
\hline & $-\mathrm{NADPH}$ & +NADPH & Ratio & -NADPH & +NADPH & Ratio & -NADPH & $+\mathrm{NADPH}$ & Ratio \\
\hline CYP1A2 & $>612$ & $>612$ & 1 & $>596$ & $>596$ & 1 & $>612$ & $>612$ & 1 \\
\hline CYP2B6 & $>612$ & $>612$ & 1 & $>596$ & $>596$ & 1 & $>612$ & $>612$ & 1 \\
\hline CYP2C8 & $>612$ & $>612$ & 1 & $>596$ & $>596$ & 1 & $>612$ & $>612$ & 1 \\
\hline CYP2C9 & $>612$ & $>612$ & 1 & $>596$ & $>596$ & 1 & $>612$ & $>612$ & 1 \\
\hline CYP2C19 & $>612$ & $>612$ & 1 & $>596$ & $>596$ & 1 & $>612$ & $>612$ & 1 \\
\hline CYP2D6 & $>612$ & $>612$ & 1 & $>596$ & $>596$ & 1 & $>612$ & $>612$ & 1 \\
\hline CYP3A4/5 (midazolam) & $>612$ & $>612$ & 1 & $>596$ & $>596$ & 1 & $>612$ & $>612$ & 1 \\
\hline CYP3A4/5 (testosterone) & $>612$ & $>612$ & 1 & $>596$ & $>596$ & 1 & $>612$ & $>612$ & 1 \\
\hline
\end{tabular}


TABLE 5

Time-dependent inhibition results for GalNAc siRNAs against P450s

\begin{tabular}{|c|c|c|c|c|c|c|c|c|c|}
\hline \multirow{3}{*}{ P450 Isoform } & \multicolumn{9}{|c|}{$\mathrm{IC}_{50}(\mu \mathrm{M})$} \\
\hline & \multicolumn{3}{|c|}{ Inclisiran } & \multicolumn{3}{|c|}{ AGT01 } & \multicolumn{3}{|c|}{ HBV02 } \\
\hline & -NADPH & +NADPH & Ratio & -NADPH & +NADPH & Ratio & -NADPH & +NADPH & Ratio \\
\hline CYP1A2 & $>612$ & $>612$ & 1 & $>18.3$ & $>18.3$ & 1 & $>20$ & $>20$ & 1 \\
\hline CYP2B6 & $>612$ & $>612$ & 1 & $>18.3$ & $>18.3$ & 1 & $>20$ & $>20$ & 1 \\
\hline CYP2C8 & $>612$ & $>612$ & 1 & $>18.3$ & $>18.3$ & 1 & $>20$ & $>20$ & 1 \\
\hline CYP2C9 & $>612$ & $>612$ & 1 & $>18.3$ & $>18.3$ & 1 & $>20$ & $>20$ & 1 \\
\hline CYP2C19 & $>612$ & $>612$ & 1 & $>18.3$ & $>18.3$ & 1 & $>20$ & $>20$ & 1 \\
\hline CYP2D6 & $>612$ & $>612$ & 1 & $>18.3$ & $>18.3$ & 1 & $>20$ & $>20$ & 1 \\
\hline CYP3A4/5 (midazolam) & $>612$ & $>612$ & 1 & $>18.3$ & $>18.3$ & 1 & $>20$ & $>20$ & 1 \\
\hline CYP3A4/5 (testosterone) & $>612$ & $>612$ & 1 & $>18.3$ & $>18.3$ & 1 & $>20$ & $>20$ & 1 \\
\hline
\end{tabular}

\section{Estimation of Total Human Liver Concentrations}

Since it is known that GalNAc-siRNA liver concentrations are much higher than the plasma concentrations in nonclinical animal models, it is expected that the liver levels are likewise going to be higher in human, although they cannot be directly measured. The EMA recommends in these cases that the liver concentration for DDI assessment should be considered as a worst-case method (European Medicines Agency, 2012). Considering this, the human relevant maximal liver concentrations were estimated from the nonclinical animal data and used for modeling DDI potential. The relationship between rat and monkey preferential liver distribution (liver $C_{\max }$ /plasma $C_{\max }$ ) is displayed in Fig. 4. The values were positively correlated with monkey liver enrichment being greater than rat liver enrichment values for all GalNAc siRNAs investigated. The mean estimated liver concentrations from the in vitro data and two approaches for modeling are displayed in Table 1. These values represent the total estimated liver concentration, and therefore are considered to be the worst-case scenario. Given that
ASGPR mediates uptake through endocytosis into the lysosomal compartment, it is expected that the free intracellular concentration available to interact with the drug-metabolizing enzymes located in the endoplasmic reticulum is much less than the total estimated liver concentration. The total liver concentration was estimated to be between 50- and 870-fold higher than the plasma concentration across the six GalNAc-siRNA conjugates used to inform the model.

\section{Clinical DDI Risk Assessment}

Evaluation of GalNAc siRNAs as Perpetrators of DDI against P450. GalNAc siRNAs did not cause in vitro induction or timedependent inhibition of P450s; therefore, clinically relevant induction or time-dependent inhibition is not expected. One GalNAc siRNA (cemdisiran) caused concentration-dependent inhibition of CYP2B6 and three GalNAc siRNAs (fitusiran, cemdisiran, and lumasiran) caused concentration-dependent inhibition of $\mathrm{CYP} 2 \mathrm{C} 8$ at supratherapeutic concentrations. Based on these observations, a clinical risk assessment

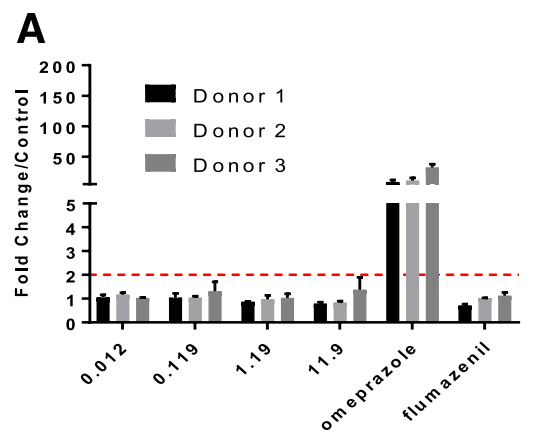

B

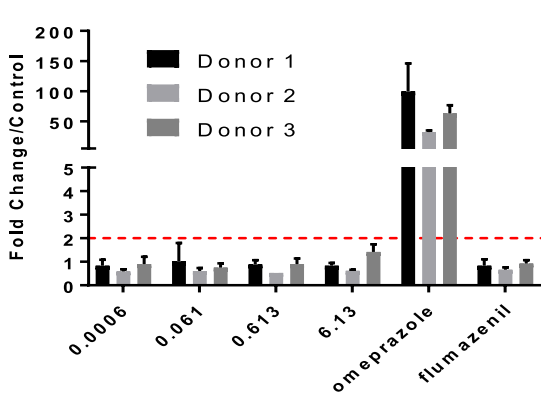

[givosiran] $\mu M$ (CYP1A2)

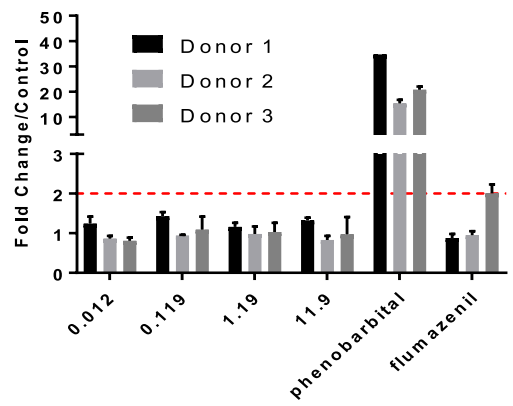

[cemdisiran] $\mu$ M (CYP2B

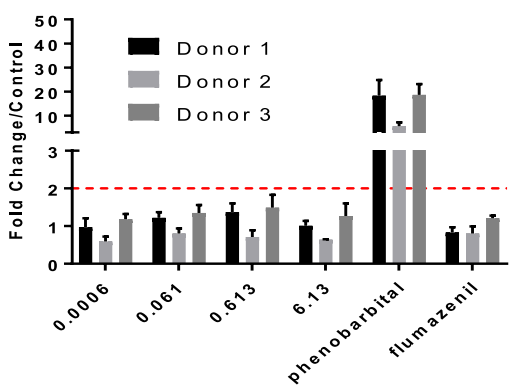

[givosiran] $\mu$ M (CYP2B 6)

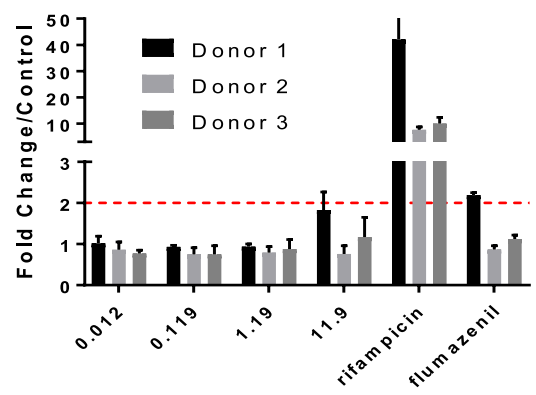

[cemdisiran] $\mu$ M (CYP3A4)

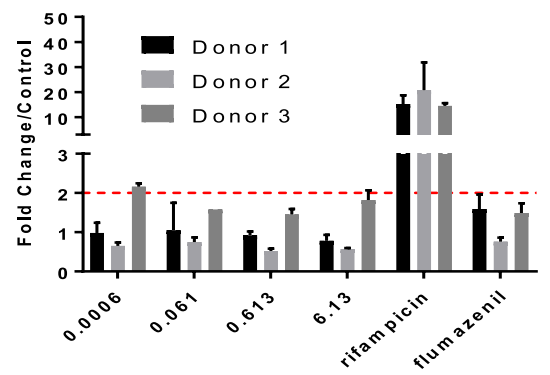

[givosiran] $\mu \mathrm{M}$ (CYP3A4)

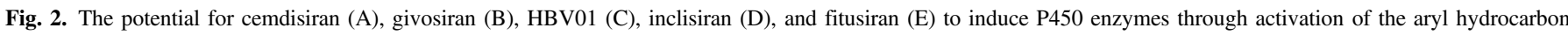

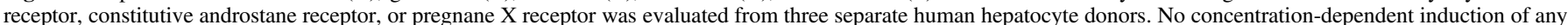
of the isoforms evaluated was observed. Concentration-dependent decreases in mRNA levels for two donors was observed after givosiran treatment. 

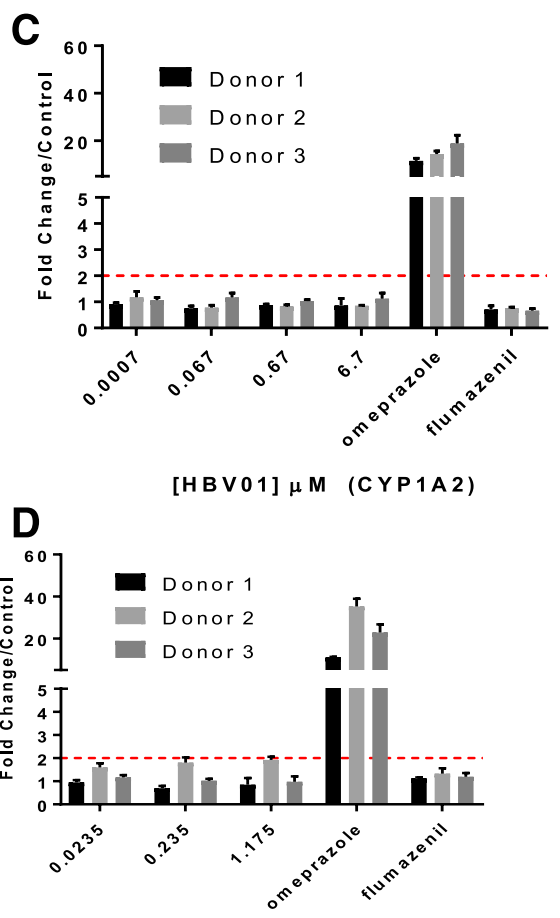

[inclisiran] $\mu M \quad$ (CYP1A2)

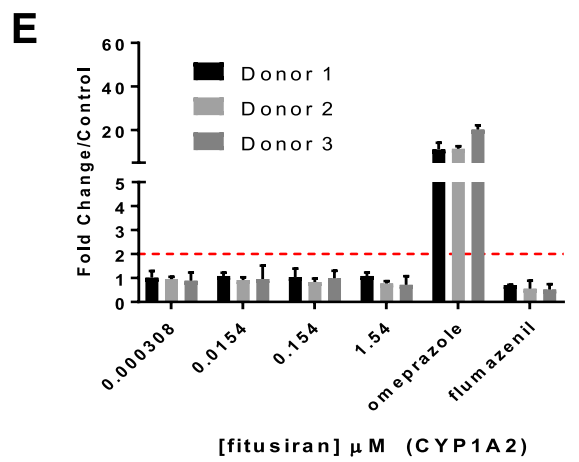

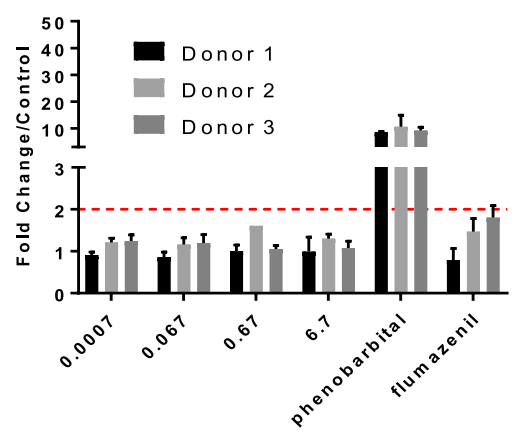

[HBV01] $\mu$ M (CYP 2B 6)

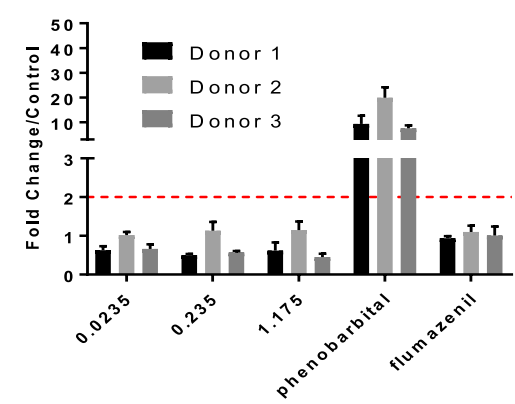

[inclisiran] $\mu M \quad($ CYP $2 B 6)$

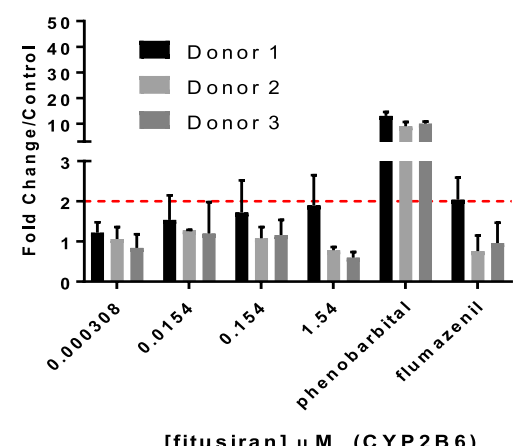

[firan ] M (CYP2B 6)

Fig. 2. Continued.

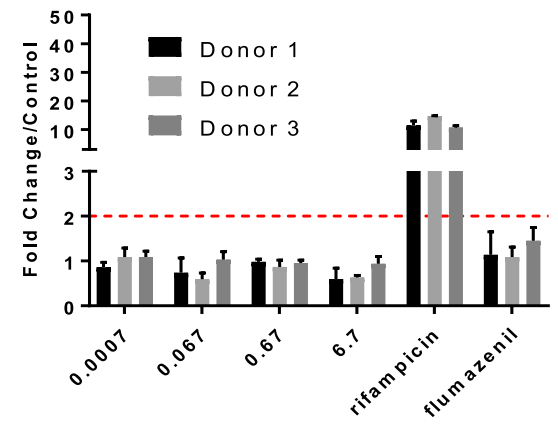

[HBV01] $\mu$ M (CYP3A4)

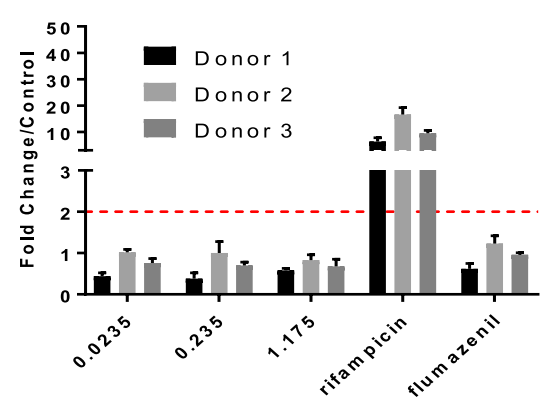

[inclisiran] $\mu M \quad(C Y P 3 A 4)$

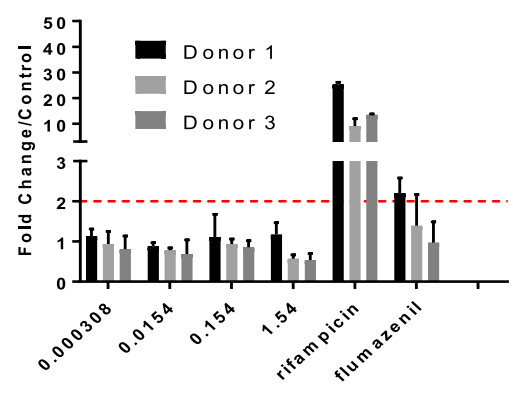

[fitusiran] $\mu M($ CY 3 A 4 ) was made for CYP2B6 and CYP2C8 inhibition potential, which was consistent with recommendations made in regulatory guidelines [EMA, Food and Drug Administration (FDA), and Pharmaceutical and Medical Devices Agency], as shown in Table 8. All of the regulatory agencies are

TABLE 6

Evaluation of metabolic stability for inclisiran in the incubation media during the induction study

\begin{tabular}{cccc}
\hline \multirow{2}{*}{ Inclisiran } & Donor & \multicolumn{2}{c}{ Percentage remaining compared to 0 h (Mean \pm S.D.; $n=3)$} \\
\cline { 3 - 4 } & & $6 \mathrm{~h}$ & $24 \mathrm{~h}$ \\
\hline$\mu M$ & & & \\
0.0245 & 1 & $99.3 \pm 2.09$ & $111 \pm 0.76$ \\
& 2 & $94.5 \pm 1.84$ & $123 \pm 2.68$ \\
0.245 & 3 & $100 \pm 1.33$ & $107 \pm 4.51$ \\
& 1 & $96.1 \pm 3.18$ & $117 \pm 1.79$ \\
& 2 & $98.3 \pm 0.83$ & $126 \pm 0.29$ \\
1.22 & 3 & $102 \pm 1.31$ & $110 \pm 3.92$ \\
& 1 & $96.8 \pm 2.90$ & $116 \pm 6.66$ \\
& 2 & $104 \pm 1.95$ & $124 \pm 7.05$ \\
& 3 & $99.3 \pm 2.69$ & $114 \pm 2.99$ \\
\hline
\end{tabular}

aligned in the cutoff criterion used for evaluating P450 inhibition potential. Namely, that clinical risk cannot be excluded if the $R_{1}$ value is $>1.02$, where $R_{1}=1+\mathrm{I} / K_{\mathrm{i}}$ and $I$ is the unbound $C_{\max }$ at steady state. Given that GalNAc siRNAs are targeted to the liver, the estimated liver concentrations were also used as a worst-case evaluation, and a cutoff of $R_{1}>1.1$ was applied. It should be noted that the regulatory guidance documents do not have recommendations for cutoff criteria when using input concentrations other than plasma. However, the highly conservative cutoff of 1.02 is recommended, with the goal of avoiding false negatives by taking into account inherent system variability and/or accumulation at the interaction site. Using estimated liver concentrations represents a conservative approach beyond using unbound plasma concentrations; therefore, the cutoff was set as 1.1, which is still below the threshold of clinically relevant inhibition (1.25). When the $R_{1}$ value is $>1.02$ (plasma) or 1.1 (liver), a second-tier approach can be used that includes modeling the potential with either a mechanistic static (net effect) or dynamic (physiologically based pharmacokinetic) model. If the second-tier model is $\geq 1.25$, then clinical evaluation would be recommended. Using the plasma concentration, the risk of clinically relevant inhibition of CYP2B6 or CYP2C8 is low. When using the liver 
TABLE 7

Confirmation of uptake into sandwich cultured human hepatocytes

\begin{tabular}{lcc}
\hline GalNAc siRNA & $\begin{array}{c}\text { Nominal Incubation } \\
\text { Concentration }\end{array}$ & $\begin{array}{c}\text { Measured Intracellular Concentration } \\
\text { (Mean } \pm \text { S.D.; } n=3 \text { ) }\end{array}$ \\
\hline \multirow{3}{*}{ Fitusiran } & $\mu M$ & $\mu M$ \\
& 0.000308 & $0.0467 \pm 0.00761$ \\
& 0.0154 & $0.181 \pm 0.0315$ \\
HBV01 & 0.154 & $1.12 \pm 0.165$ \\
& 1.54 & $5.08 \pm 1.82$ \\
& 0.000665 & $0.0044 \pm 0.000734$ \\
Givosiran & 0.00665 & $0.0271 \pm 0.00825$ \\
& 0.0665 & $0.687 \pm 0.283$ \\
& 6.65 & $2.95 \pm 1.5$ \\
Inclisiran & 0.000613 & $0.000923 \pm 0.0000883$ \\
& 0.00613 & $0.0496 \pm 0.0081$ \\
Cemdisiran & 0.613 & $1.34 \pm 0.15$ \\
& 6.13 & $3.25 \pm 1.53$ \\
& 0.0245 & $0.0571 \pm 0.00788$ \\
& 0.245 & $0.288 \pm 0.038$ \\
& 1.22 & $0.734 \pm 0.135$ \\
& 0.0119 & $0.191 \pm 0.027$ \\
& 0.119 & $1.04 \pm 0.0235$ \\
& 1.19 & $3.90 \pm 0.368$ \\
& 11.9 & $10.1 \pm 2.35$ \\
\hline
\end{tabular}

concentration, the potential for clinically relevant inhibition of CYP2C8 by fitusiran and cemdisiran could not be excluded by the $R_{1}$ equation; therefore, mechanistic static modeling was conducted. The substrate used in the modeling was cerivastatin $\left(F_{\mathrm{m}}=0.82\right)$, which is more sensitive toward CYP2C8 than other substrates (i.e., repaglinide, $F_{\mathrm{m}}=$ $0.49-0.63)$. Using this approach, the potential for clinically relevant DDI of CYP2C8 is low $(R<1.25)$ and no clinical interaction studies are warranted.

\section{Givosiran as a P-gp Inhibitor}

The current FDA and Pharmaceutical and Medical Devices Agency draft DDI guidance documents propose a cutoff based only on orally administered drugs and recommend using $I_{\text {gut }} / \mathrm{IC}_{50}>10$. The route of administration for GalNAc siRNAs is through subcutaneous injection, thus the potential for GalNAc siRNAs to mediate gut level DDI on P-gp is low. For this reason, the cutoff of $I_{1} / \mathrm{IC}_{50} \geq 0.03$ proposed by Ellens et al. (2013) and Lee et al. (2017) was used to determine whether in vitro inhibition of P-gp by givosiran could be clinically relevant. Based on this analysis, the likelihood for givosiran to inhibit P-gp in the clinic is very low (area under the concentration-time curve ratio $=1.0$ ).

\section{Discussion}

Regulatory authorities encourage thorough investigation of DDI potential during drug development since it can lead to adverse clinical effects or reduction in efficacy. These investigations are often carried out in vitro and typically focus on the major drug-metabolizing enzymes (P450s and UDP-glucuronosyltransferases) and transporters, where clinically relevant DDI has been observed. There is currently no guidance addressing DDI potential of novel therapeutics such as oligonucleotides. Therefore, recommendations made for small molecules are often adhered to during their development. Years of experience have enabled the recommendation that DDI of protein therapeutics can be determined on a case-by-case basis (Huang et al., 2010), since DDI potential is low with the exception of cytokine modulators (Kenny et al., 2013). The rapid development of novel therapeutics containing oligonucleotides has resulted in many clinical studies over the last 30 years when the first ASO, fomivirsen, was approved. An additional six ASOs have been approved, which include pegaptanib (in 2004), mipomersen (in 2013), eteplirsen, defibrotide, nusinersen (in 2016), and inotersen (in 2018). During this time frame no significant pharmacokinetic DDIs have been reported (Geary et al., 2002, 2006; Yu et al., 2009; Li et al., 2014). The first siRNA, ONPATTRO (patisiran), approved by the FDA in 2018, employs a different dosing route and delivery mechanism than the current generation of GalNAc siRNAs that are the focus of this paper.
A

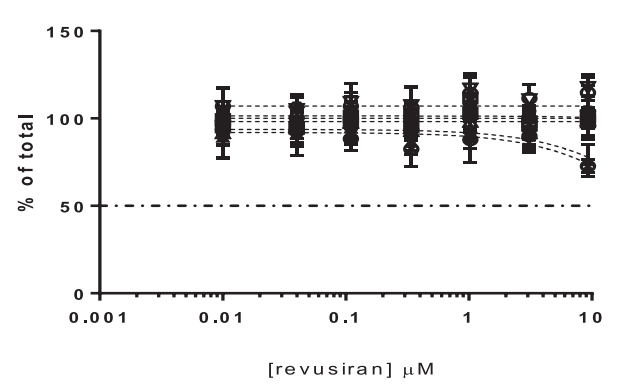

C

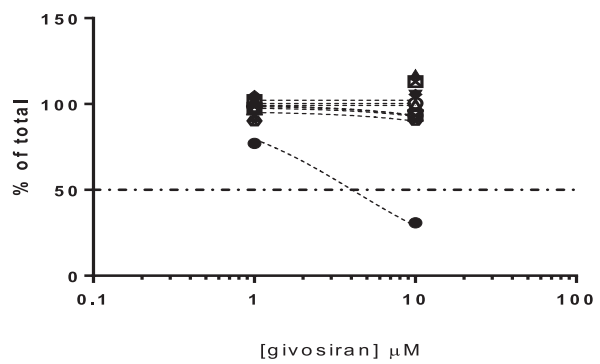

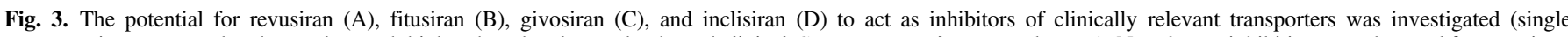

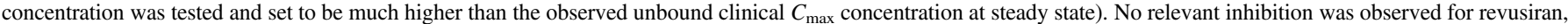
fitusiran, and inclisiran against any of the transporters evaluated. Givosiran resulted in concentration-dependent decreases in P-gp activity.

-. $M D R 1$

- BCRP

- - BSEP

- MAte1

- mate2-k

ロ. OATP1B 1

A- OATP1B3

7. OAT1

- oAt3

- OCT1

- oct2

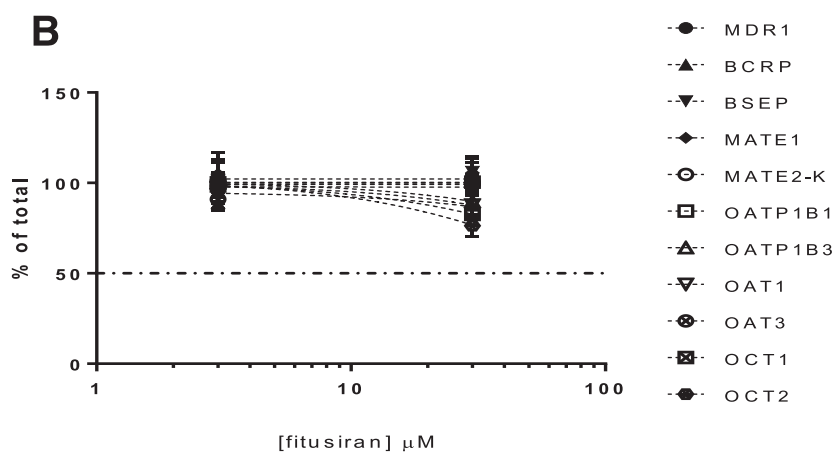

D

- $M D R 1-1 C^{2}=4.2 \mu M$

- BCRP

- BSEP

- mate1

- mate2-K

ㅁ. OATP1B 1

$\triangle$ - OATP1B3

ק- OAT1

- oAt3

- OCT1

- ост2

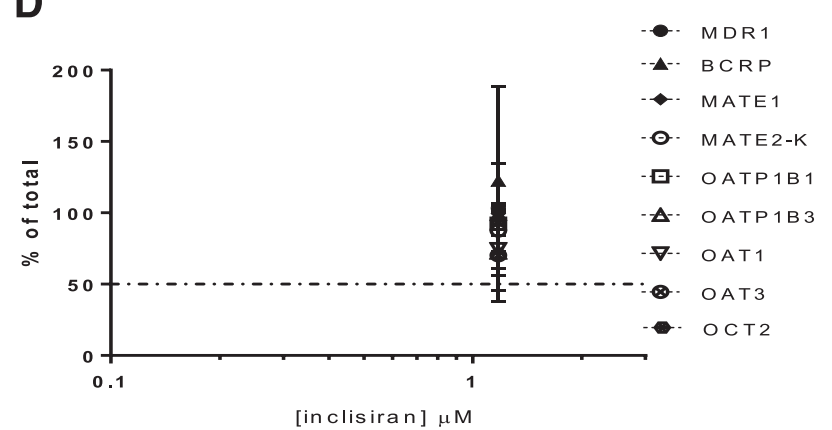




\section{Liver to plasma ratio}

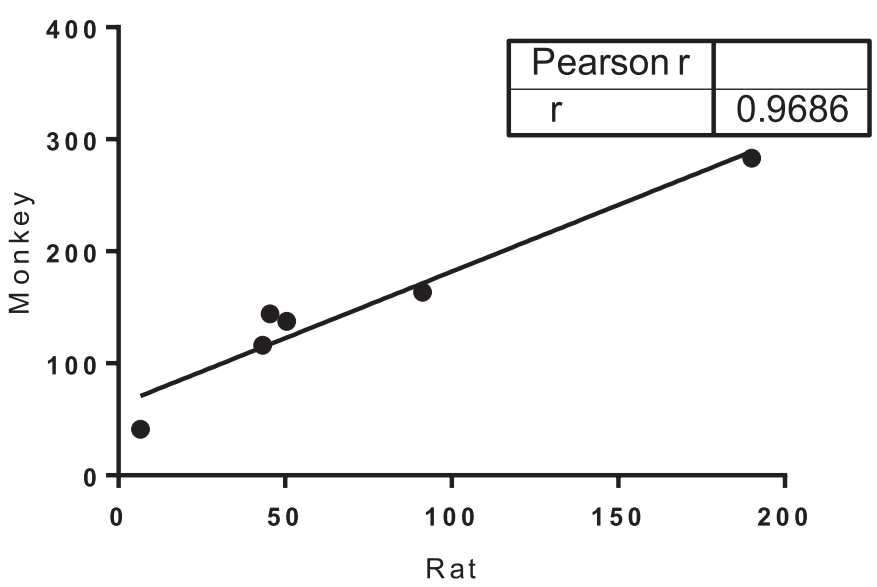

Fig. 4. Correlation analysis of liver enrichment observed in rat vs. cynomolgus monkey. In this plot the liver enrichment represents the preferential distribution of GalNAc siRNAs into liver when compared with plasma $\left(C_{\max } \operatorname{liver} / C_{\max }\right.$ plasma); since this is a ratio, it is unitless.

Considering the mechanism of action, disposition, and molecular size, it is unlikely that GalNAc siRNAs would interact with drugmetabolizing enzymes and transporters. They are highly soluble in water and negatively charged at physiologic pH. ASGPR-mediated uptake efficiently delivers GalNAc siRNA to the liver; thus, solute carrier transporters are unlikely to contribute to their active uptake. In nonclinical models (rat and monkey), renal clearance is not a major route of elimination of GalNAc siRNAs (less than $25 \%$ of total clearance), suggesting that the role of renal transporters is likely minor. Metabolite identification studies indicate that GalNAc siRNAs are metabolized to shortmers by exo- and endonucleases. GalNAc siRNAs do not induce cytokines in vitro or in vivo; therefore, modulation of drug-metabolizing activity through this mechanism is unlikely.

Reaction phenotyping of seven GalNAc siRNAs confirmed that they are not substrates of P450 enzymes, including CYP1A2, 2A6, 2B6, 2C8, 2C9, 2C19, 2D6, 3A4, and 3A5. Therefore, clinical interactions with inhibitors or inducers of P450 enzymes would not be expected. None of the nine GalNAc siRNAs tested caused any time-dependent inhibition of the major P450 enzymes, including CYP1A2, 2A6, 2B6, 2C8, 2C9, 2C19, 2D6, 3A4, and 3A5. Mild direct inhibition was observed for CYP2B6 with cemdisiran $\left(K_{\mathrm{i}}=292 \mu \mathrm{M}\right)$ and for CYP2C8 with cemdisiran $\left(K_{\mathrm{i}}=112 \mu \mathrm{M}\right)$, fitusiran $\left(K_{\mathrm{i}}=28 \mu \mathrm{M}\right)$, and lumasiran $\left(K_{\mathrm{i}}=\right.$ $208 \mu \mathrm{M})$. A recent publication by Sekisui XenoTech postulated that the phosphorothioate linkages contained within the evaluated ASOs could be the reason for the observed direct inhibition of $\mathrm{P} 450$ in HLMs, which tended to be rank ordered as CYP1A2 $\approx$ CYP2C8 $>$ CYP2B6 (Kazmi et al., 2018). The authors demonstrated test system dependency, whereby inhibition studies carried out using a more physiologically relevant hepatocyte model resulted in no inhibition or reduced extents of inhibition. Unlike ASO molecules, which contain at least 12 phosphorothioate linkages, GalNAc siRNAs contain a maximum of six phosphorothioate linkages. To evaluate the clinical relevance of CYP2B6 or CYP2C8 in vitro inhibition, a risk assessment using the decision trees presented in regulatory DDI guidance documents was performed. Since GalNAc siRNAs are efficiently delivered to the hepatocytes via ASGPR, the liver concentration is anticipated to be much higher than plasma. However, as previously mentioned, the free intracellular concentration available to inhibit P450 enzymes on the endoplasmic reticulum is likely to be much lower than the total liver concentration. A worst-case assessment of DDI potential was made by inputting estimated total liver concentrations in addition to plasma to project the clinical outcome. Based on this analysis, no clinical DDI evaluation would be triggered $(R<1.25)$. It is possible that conducting inhibition studies using a more physiologically representative model such as hepatocytes, similarly to ASOs, would yield lower extents of inhibition. However, since clinically relevant inhibition is not expected, these studies were not conducted.

There was no modulation of drug-metabolizing enzymes observed during in vitro induction studies characterizing endpoints of the aryl hydrocarbon receptor, constitutive androstane receptor, and pregnane $\mathrm{X}$ receptor. Thus, clinical interactions due to induction of drugmetabolizing enzymes by GalNAc siRNAs are unlikely. Taken together, the likelihood for GalNAc siRNAs to perpetrate DDI against P450 substrates is unlikely.

Transporters can drive intracellular concentrations and mediate clinically relevant DDIs; thus, regulatory agencies, such as the EMA, FDA, and Pharmaceutical and Medical Devices Agency, recommend that all new chemical entities be evaluated as substrates or inhibitors of transporters. In vitro investigations can be prioritized based on knowledge of the new chemical entity as well as the physiochemical properties. For example, compounds with high solubility or permeability do not need to be evaluated as substrates of P-gp and BCRP and renal transporters only need to be evaluated if renal excretion is a major elimination pathway (European Medicines Agency, 2012; Food and Drug Administration, 2017). Inhibition of P-gp and BCRP should also be considered only for those molecules administered orally. The rationale for this is because clinically relevant DDIs are more likely to occur in the gastrointestinal tract, where inhibitor concentrations would be much higher than plasma, and therefore can achieve a high enough level to inhibit intestinal P-gp. A review of clinical DDI studies with common P-gp substrates (digoxin, dabigatran, loperamide, and apixaban) yielded 249 total clinical interaction studies. Of these, 130 studies were positive for inhibition and all precipitants were dosed orally. Only 10 clinical DDI studies have been conducted to date with perpetrators dosed subcutaneously (five) or intravenously (five), and none of these studies resulted in relevant clinical inhibition. Clinical P-gp DDI studies

TABLE 8

Assessment of the clinical drug-drug interaction potential based on reversible inhibition of metabolizing enzymes and cutoff criteria from regulatory guidance

\begin{tabular}{|c|c|c|c|c|c|c|c|c|}
\hline \multirow{2}{*}{ GalNAc siRNA } & \multirow{2}{*}{ Enzyme } & \multirow{2}{*}{$K_{\mathrm{i}}$} & \multicolumn{3}{|c|}{ Total Plasma $C_{\text {max,ss }}\left(\right.$ Observed Clinical Concentration at Therapeutic Dose) ${ }^{a}$} & \multicolumn{2}{|c|}{$R_{1}$} & \multirow{2}{*}{ Mechanistic Static Model (Liver) } \\
\hline & & & EMA & FDA & PMDA & Plasma & Liver & \\
\hline & & & $\mu M$ & $\mu M$ & $\mu M$ & & & \\
\hline Cemdisiran & CYP2B6 & 292 & $5.83(0.048)$ & $5.83(0.048)$ & $5.83(0.048)$ & 1.00 & 1.04 & 1.03 \\
\hline Fitusiran & CYP2C8 & 28 & $0.56(0.0092)$ & $0.56(0.0092)$ & $0.56(0.0092)$ & 1.00 & 1.15 & 1.12 \\
\hline Cemdisiran & CYP2C8 & 112 & $2.24(0.048)$ & $2.24(0.048)$ & $2.24(0.048)$ & 1.00 & 1.10 & 1.08 \\
\hline Lumasiran & CYP2C8 & 208 & $4.16(0.061)$ & $4.16(0.061)$ & $4.16(0.061)$ & 1.00 & 1.03 & 1.03 \\
\hline
\end{tabular}

$C_{\max , s s}, C_{\max }$ at steady state; PMDA, Pharmaceutical and Medical Devices Agency.

${ }^{a}$ Total plasma $C_{\text {max,ss }}$ required to cause clinically relevant inhibition based on DDI guidance criteria; cutoff $=R=1+50 \times[I] / K_{\mathrm{i}}$, where $I$ is the unbound $C_{\text {max,ss }}, R \geq 2.0$, and $F_{\mathrm{u}}=1$. 
with drugs administered subcutaneously were limited to glucagon-like peptide-1 receptor agonists (albiglutide, dulaglutide, lixisenatide, and semaglutide) because of an on-target delayed gastric emptying, which could alter the gastrointestinal absorption kinetics of coadministered therapies. The route of administration for GalNAc siRNAs is through subcutaneous injection, thus the potential for GalNAc siRNAs to mediate gut level DDI on P-gp is low. The likelihood for givosiran to result in clinically meaningful inhibition of P-gp was determined to be low when considering the in vitro-derived $\mathrm{IC}_{50}$ value. Current data across GalNAc siRNAs demonstrate low potential for inhibition interactions with transporters. Furthermore, GalNAc siRNAs were determined not to be substrates of clinically relevant transporters. Overall, these data confirm that the likelihood for clinically relevant interactions with transporters by GalNAc siRNAs is low.

The totality of data confirms that chemical inhibition or induction of drug-metabolizing enzymes and transporters mediated by GalNAc siRNAs is highly unlikely. The potential for pharmacologically mediated DDI should be evaluated by understanding the mechanism of action and downstream or upstream effects of target gene knockdown. A weight-of-evidence approach for including/excluding clinical DDI investigation dependent on the properties of the siRNA and the pharmacology of the target is recommended. To reduce off-target effects, siRNA sequences are carefully designed, with the first step employing algorithms aimed to reduce the probability of perfect matches and hybridization with RNAs for genes other than the target followed by a strategy to mitigate off-target activity. In general, based on this large data set, in the absence of any pathway-mediated DDI potential, it can be concluded that GalNAc siRNAs are unlikely to be a victim or perpetrator of DDIs and in vitro or clinical investigations are not warranted.

\section{Acknowledgments}

The authors thank Bahru Habtemariam, Martin Maier, Vasant Jadhav, Gabriel Robbie, and Sara Nochur for thoughtful discussions and critical review of the manuscript.

\section{Authorship Contributions}

Participated in research design: Ramsden, Wu, Zerler, Iqbal, Jiang, Clausen, Chong.

Conducted experiments: Aluri, Gu, Dennin, Kim.

Performed data analysis: Ramsden.

Wrote or contributed to the writing of the manuscript: Ramsden, Wu, Chong.

\section{References}

Bon C, Hofer T, Bousquet-Mélou A, Davies MR, and Krippendorff BF (2017) Capacity limits of asialoglycoprotein receptor-mediated liver targeting. MAbs 9:1360-1369.

Cheng Y and Prusoff WH (1973) Relationship between the inhibition constant $\left(K_{I}\right)$ and the concentration of inhibitor which causes 50 per cent inhibition $\left(I_{50}\right)$ of an enzymatic reaction. Biochem Pharmacol 22:3099-3108.

Elbashir SM, Harborth J, Lendeckel W, Yalcin A, Weber K, and Tuschl T (2001) Duplexes of 21-nucleotide RNAs mediate RNA interference in cultured mammalian cells. Nature 411 494-498.

Ellens H, Deng S, Coleman J, Bentz J, Taub ME, Ragueneau-Majlessi I, Chung SP, Heredi-Szabo K, Neuhoff S, Palm J, et al. (2013) Application of receiver operating characteristic analysis to refine the prediction of potential digoxin drug interactions. Drug Metab Dispos 41:1367-1374.

European Medicines Agency (2012) Guideline on the Investigation of Drug Interactions, Committee for Medicinal Products for Human Use, European Medicines Agency, London.

Fahmi OA, Hurst S, Plowchalk D, Cook J, Guo F, Youdim K, Dickins M, Phipps A, Darekar A, Hyland R, et al. (2009) Comparison of different algorithms for predicting clinical drug-drug interactions, based on the use of CYP3A4 in vitro data: predictions of compounds as precipitants of interaction. Drug Metab Dispos 37:1658-1666.

Fitzgerald K, Kallend D, and Simon A (2017) A highly durable RNAi therapeutic inhibitor of PCSK9. N Engl J Med 376:e38.

Food and Drug Administration (2017) In Vitro Metabolism- and Transporter-Mediated Drug-Drug Interaction Studies Guidance for Industry, U.S. Department of Health and Human Services Food and Drug Administration Center for Drug Evaluation and Research, Silver Spring, MD.

Foster DJ, Brown CR, Shaikh S, Trapp C, Schlegel MK, Qian K, Sehgal A, Rajeev KG, Jadhav V, Manoharan M, et al. (2018) Advanced siRNA designs further improve in vivo performance of GalNAc-siRNA conjugates. Mol Ther 26:708-717.

Geary RS, Bradley JD, Watanabe T, Kwon Y, Wedel M, van Lier JJ, and VanVliet AA (2006) Lack of pharmacokinetic interaction for ISIS 113715, a 2'-O-methoxyethyl modified antisense oligonucleotide targeting protein tyrosine phosphatase 1B messenger RNA, with oral antidiabetic compounds metformin, glipizide or rosiglitazone. Clin Pharmacokinet 45:789-801.

Geary RS, Henry SP, and Grillone LR (2002) Fomivirsen: clinical pharmacology and potential drug interactions. Clin Pharmacokinet 41:255-260.

Grimm SW, Einolf HJ, Hall SD, He K, Lim HK, Ling KH, Lu C, Nomeir AA, Seibert E, Skordos $\mathrm{KW}$, et al. (2009) The conduct of in vitro studies to address time-dependent inhibition of drugmetabolizing enzymes: a perspective of the pharmaceutical research and manufacturers of America. Drug Metab Dispos 37:1355-1370.

Grubbs FE (1950) Sample Criteria for Testing Outlying Observations. Ann. Math. Statist. 21(1) 27-58.

Huang SM, Zhao H, Lee JI, Reynolds K, Zhang L, Temple R, and Lesko LJ (2010) Therapeutic protein-drug interactions and implications for drug development. Clin Pharmacol Ther 87:497-503. Humphreys SC, Thayer MB, Lade JM, Wu B, Sham K, Basiri B, Hao Y, Huang X, Smith R, and Rock BM (2019) Plasma and liver protein binding of GalNAc conjugated siRNA. Drug Metab Dispos DOI: 10.1124/dmd.119.086967 [published ahead of print].

Janas MM, Zlatev I, Liu J, Jiang Y, Barros SA, Sutherland JE, Davis WP, Liu J, Brown CR, Liu X, et al. (2019) Safety evaluation of 2'-deoxy-2'-fluoro nucleotides in GalNAc-siRNA conjugates. Nucleic Acids Res 47:3306-3320.

Kalvass JC, Maurer TS, and Pollack GM (2007) Use of plasma and brain unbound fractions to assess the extent of brain distribution of 34 drugs: comparison of unbound concentration ratios to in vivo P-glycoprotein efflux ratios. Drug Metab Dispos 35:660-666.

Kazmi F, Yerino P, McCoy C, Parkinson A, Buckley DB, and Ogilvie BW (2018) An assessment of the in vitro inhibition of cytochrome P450 enzymes, UDP-glucuronosyltransferases, and transporters by phosphodiester- or phosphorothioate-linked oligonucleotides. Drug Metab Dispos 46:1066-1074.

Kenny JR, Liu MM, Chow AT, Earp JC, Evers R, Slatter JG, Wang DD, Zhang L, and Zhou H (2013) Therapeutic protein drug-drug interactions: navigating the knowledge gaps-highlights from the 2012 AAPS NBC Roundtable and IQ Consortium/FDA workshop. AAPS J 15:933-940. Kim D and Rossi J (2008) RNAi mechanisms and applications. Biotechniques 44:613-616.

Landesman Y, Svrzikapa N, Cognetta A III, Zhang X, Bettencourt BR, Kuchimanchi S, Dufault K, Shaikh S, Gioia M, Akinc A, et al. (2010) In vivo quantification of formulated and chemically modified small interfering RNA by heating-in-Triton quantitative reverse transcription polymerase chain reaction (HIT qRT-PCR). Silence 1:16

Lee SC, Arya V, Yang X, Volpe DA, and Zhang L (2017) Evaluation of transporters in drug development: Current status and contemporary issues. Adv Drug Deliv Rev 116:100-118.

Li Z, Hard ML, Grundy JS, Singh T, von Moltke LL, and Boltje I (2014) Lack of clinical pharmacodynamic and pharmacokinetic drug-drug interactions between warfarin and the antisense oligonucleotide mipomersen. J Cardiovasc Pharmacol 64:164-171.

Nair JK, Attarwala H, Sehgal A, Wang Q, Aluri K, Zhang X, Gao M, Liu J, Indrakanti R, Schofield $S$, et al. (2017) Impact of enhanced metabolic stability on pharmacokinetics and pharmacodynamics of GalNAc-siRNA conjugates. Nucleic Acids Res 45:10969-10977.

Nair JK, Willoughby JL, Chan A, Charisse K, Alam MR, Wang Q, Hoekstra M, Kandasamy P, Kel'in AV, Milstein S, et al. (2014) Multivalent $N$-acetylgalactosamine-conjugated siRNA localizes in hepatocytes and elicits robust RNAi-mediated gene silencing. I Am Chem Soc 136:16958-16961.

Pasi KJ, Rangarajan S, Georgiev P, Mant T, Creagh MD, Lissitchkov T, Bevan D, Austin S, Hay CR, Hegemann I, et al. (2017) Targeting of antithrombin in hemophilia A or B with RNA therapy. N Engl J Med 377:819-828.

Pharmaceutical and Medical Devices Agency (2018) Guideline of drug interaction studies for drug development and appropriate provision of information. https://www.pmda.go.jp/files/000228122.pdf.

Ramsden D, Tweedie DJ, Chan TS, Taub ME, and Li Y (2014) Bridging in vitro and in vivo metabolism and transport of faldaprevir in human using a novel cocultured human hepatocyte system, HepatoPac. Drug Metab Dispos 42:394-406.

Riccardi K, Lin J, Li Z, Niosi M, Ryu S, Hua W, Atkinson K, Kosa RE, Litchfield J, and Di L (2017) Novel method to predict in vivo liver-to-plasma $K_{\text {puu }}$ for OATP substrates using suspension hepatocytes. Drug Metab Dispos 45:576-580.

Sane RS, Ramsden D, Sabo JP, Cooper C, Rowland L, Ting N, Whitcher-Johnstone A and Tweedie DJ (2016) Contribution of major metabolites toward complex drug-drug interactions of deleobuvir: in vitro predictions and in vivo outcomes. Drug Metab Dispos 44:466-475.

Schlegel MK, Foster DJ, Kel'in AV, Zlatev I, Bisbe A, Jayaraman M, Lackey JG, Rajeev KG, Charissé K, Harp J, et al. (2017) Chirality dependent potency enhancement and structural impact of glycol nucleic acid modification on siRNA. J Am Chem Soc 139:8537-8546.

Schmittgen TD and Livak KJ (2008) Analyzing real-time PCR data by the comparative $\mathrm{C}_{\mathrm{T}}$ method. Nat Protoc 3:1101-1108.

Shemesh CS, Yu RZ, Warren MS, Liu M, Jahic M, Nichols B, Post N, Lin S, Norris DA, Hurh E, et al. (2017) Assessment of the drug interaction potential of unconjugated and $\mathrm{GalNAc}_{3}$-conjugated 2'-MOE-ASOs. Mol Ther Nucleic Acids 9:34-47.

Shen X and Corey DR (2018) Chemistry, mechanism and clinical status of antisense oligonucleotides and duplex RNAs. Nucleic Acids Res 46:1584-1600.

Soutschek J, Akinc A, Bramlage B, Charisse K, Constien R, Donoghue M, Elbashir S, Geick A, Hadwiger P, Harborth J, John M, et al. (2004) Therapeutic silencing of an endogenous gene by systemic administration of modified siRNAs. Nature 432:173-178.

Weigel PH and Yik JH (2002) Glycans as endocytosis signals: the cases of the asialoglycoprotein and hyaluronan/chondroitin sulfate receptors. Biochim Biophys Acta 1572:341-363.

Yonezawa A and Inui K (2011) Importance of the multidrug and toxin extrusion MATE/SLC47A family to pharmacokinetics, pharmacodynamics/toxicodynamics and pharmacogenomics. $\mathrm{Br} J$ Pharmacol 164:1817-1825.

Yu RZ, Geary RS, Flaim JD, Riley GC, Tribble DL, vanVliet AA, and Wedel MK (2009) Lack of pharmacokinetic interaction of mipomersen sodium (ISIS 301012), a 2'-O-methoxyethyl modified antisense oligonucleotide targeting apolipoprotein B-100 messenger RNA, with simvastatin and ezetimibe Clin Pharmacokinet 48:39-50.

Zimmermann TS, Karsten V, Chan A, Chiesa J, Boyce M, Bettencourt BR, Hutabarat R, Nochur S Vaishnaw A, and Gollob J (2017) Clinical proof of concept for a novel hepatocyte-targeting GalNAc-siRNA conjugate. Mol Ther 25:71-78.

Address correspondence to: Diane Ramsden, Drug Metabolism and Pharmacokinetics, Early Development, Alnylam Pharmaceuticals, 300 Third Street, Cambridge, MA 02142. E-mail: dramsden@alnylam.com 